TI 2007-069/2

Tinbergen Institute Discussion Paper

Idiosyncratic Labour Income Risk and Aggregate Consumption: An Unobserved Component Approach

Lorenzo Pozzi 


\section{Tinbergen Institute}

The Tinbergen Institute is the institute for economic research of the Erasmus Universiteit Rotterdam, Universiteit van Amsterdam, and Vrije Universiteit Amsterdam.

Tinbergen Institute Amsterdam

Roetersstraat 31

1018 WB Amsterdam

The Netherlands

Tel.: $\quad+31(0) 205513500$

Fax: $\quad+31(0) 205513555$

Tinbergen Institute Rotterdam

Burg. Oudlaan 50

3062 PA Rotterdam

The Netherlands

Tel.: $\quad+31(0) 104088900$

Fax: $\quad+31(0) 104089031$

Most TI discussion papers can be downloaded at http:/ /www.tinbergen.nl. 


\title{
Idiosyncratic labour income risk and aggregate consumption: an unobserved component approach*
}

\author{
Lorenzo Pozzi ${ }^{\dagger}$
}

September 2007

\begin{abstract}
We investigate the importance of aggregate and consumer-specific or idiosyncratic labour income risk for aggregate consumption changes in the US over the period 1952-2001. Theoretically, the effect of labour income risk on consumption changes is decomposed into an aggregate and into an idiosyncratic part. Empirically, aggregate risk is modelled through a GARCH process on aggregate labour income shocks and individual risk is modelled as an unobserved component and obtained through Kalman filtering. Our results suggest that aggregate labour income risk explains a negligible fraction of the variance of aggregate consumption changes. A more important part of aggregate consumption changes is explained by the unobserved component. The interpretation of this component as reflecting idiosyncratic labour income risk is supported by the finding that it is negatively affected by received consumer transfers. Idiosyncratic labour income risk thus matters for the aggregate economy.

JEL Classification: E21

Keywords: labour income uncertainty, consumption, precaution, state space models, GARCH errors, unobserved component, Bayesian
\end{abstract}

\footnotetext{
${ }^{*}$ Work on this paper was started while I was visiting the National Bank of Belgium. I am grateful to Raf Wouters for supervision. The paper has benefited from comments by Gerdie Everaert and by participants at the 2005 IUAP Conference on Economic Policy, Growth and Business Cycles, Université Catholique de Louvain and Ghent University. It was presented at the 2006 North American Summer Meeting of the Econometric Society, University of Minnesota, Minneapolis, US.

${ }^{\dagger}$ Tinbergen Institute and Department of Economics, Erasmus University Rotterdam, Rotterdam, the Netherlands. E-mail: pozzi@few.eur.nl. Websites: www.few.eur.nl, www.tinbergen.nl.
} 


\section{Introduction.}

In this paper we investigate the effects of labour income risk on aggregate consumption changes using quarterly data for the US over the period 1952-2001. The contribution of this paper to the literature is threefold.

First, using the theoretical results of Caballero (1990) as a starting point, we present a theoretical framework in which the effect of labour income risk on the change in aggregate consumption is decomposed into two parts: the impact of aggregate labour income risk and the impact of consumer-specific or idiosyncratic labour income risk. ${ }^{1}$ This decomposition is useful because limiting income risk to aggregate income risk is too restrictive. The reason is that the variance of aggregate labour income is low. As a result, in permanent income models with time-separable preferences, the magnitude of the average growth in consumption can only be explained by values of risk aversion that are much higher than what is widely believed (see Gourinchas and Parker 2001). Another reason is that some authors argue that there is no theoretical a priori justification (e.g. Deaton 1992, p.37) or empirical evidence (e.g. Banks et al. 2001) to suggest that risk pooling mechanisms that effectively eliminate individual-specific labour income risk actually do exist.

Second, to complement studies that use a micro-based approach ${ }^{2}$, we follow a pure aggregate time series approach. Aggregate labour income risk is estimated through a GARCH process on aggregate labour income shocks. Idiosyncratic income risk is modelled as an unobserved component and estimated through Kalman filtering techniques. The identification of the unobserved component is based on the use of two determinants of income risk suggested in the literature: (government) transfers received by consumers and the business cycle. The main contribution of the paper is that we investigate whether changes in the amount of transfers received by consumers affect the unobserved component and the change in consumption. From the papers by Hubbard et al. (1995) and Engen and Gruber (2001), we know that transfers provided by the social security system (e.g. health and unemployment insurance) may reduce idiosyncratic labour income risk by providing insurance against bad draws of labour income. Pozzi (2007) documents the impact on aggregate consumption growth of the growth rate in government transfers in US data and explains

\footnotetext{
${ }^{1}$ See Banks et al. (2001) for a comparable decomposition at the individual/cohort level but without the possibility to aggregate analytically since preferences are of the constant relative risk aversion type.

${ }^{2}$ The use of micro-based uncertainty measures has certain drawbacks that can be avoided by following an aggregate approach. First, there is the small length of the available time series. Second, decomposing these measures into an aggregate and an idiosyncratic part is not straightforward. Third, the use of these measures can be problematic in the presence of measurement errors or "self-selection" problems (see Attanasio 1999 for a discussion).
} 
it with a buffer stock model containing an explicit government transfer policy that reduces the variance of idiosyncratic labour income. As far as the business cycle is concerned, Carroll (1992) notes that "the most drastic fluctuations in household income are those associated with spells of unemployment". We investigate whether changes in the unemployment rate affect the unobserved component and therefore the change in aggregate consumption. There is a literature that investigates whether idiosyncratic labour income risk shows cyclical behaviour (see Storesletten et al. 2004, Parker and Preston 2005, and Primiceri and van Rens 2004). We compare the results of our macro-based time series approach to the results of these papers that use micro data.

Third, we use a Bayesian approach to parameter estimation. A Bayesian approach allows us to incorporate prior knowledge into our estimations. Priors are also useful to estimate GARCH effects in state space systems in the presence of outliers in the data. Existing procedures to detect and correct for outliers in GARCH models (see e.g. Franses and van Dijk 1999) cannot be applied in a state space context.

Our results suggest that aggregate labour income risk explains only a negligible fraction of the variance of aggregate consumption changes. The unobserved component explains a more important part of consumption changes. This component can be interpreted as idiosyncratic labour income risk since it is negatively affected by the trend in transfers received by consumers (i.e. transfers can be considered a proxy for idiosyncratic labour income risk). This suggests that idiosyncratic labour income risk matters for the aggregate economy. We argue that, from the eighties onward, the trend change in transfers received by consumers can explain low frequency movements in consumption changes. We find no hard evidence that the unobserved component is driven by the change in the unemployment rate however. We then extend the model by adding rule-of-thumb consumers who base their consumption decisions on current income. While the existence of ruleof-thumb consumers may be due to liquidity constraints (see e.g. Campbell and Mankiw 1990) or myopia (see e.g. Flavin 1985) in this paper we emphasize a precaution-based explanation of excess sensitivity. Estimation of the extended model suggests that our results are robust when excess sensitivity of consumption to anticipated disposable income is taken into account.

The paper is structured as follows. In section 2 we present a consumption model with timevarying aggregate and time-varying idiosyncratic labour income risk. In section 3 we present our basic empirical specification which is put into state space form to estimate the unobserved component. We discuss the Bayesian estimation of the unknown parameters in the model. In section 4 we present the estimation results for our basic model. In section 5 we investigate whether 
our conclusions are affected by the introduction of rule-of-thumb consumers in the model. Section 6 concludes.

\section{A consumption model with time-varying aggregate and time-varying idiosyncratic labour income risk.}

In this section we derive an expression for the change in aggregate private consumption that takes into account uncertainty with respect to aggregate labour income and uncertainty with respect to the idiosyncratic component of labour income. The latter type of risk is present because insurance markets are assumed to be incomplete (i.e. there is no risk pooling across consumers). The model uses the results of Caballero (1990) in a setting where consumers are heterogeneous in the sense that they experience different income draws. As a result, given the absence of insurance mechanisms, consumption trajectories and wealth levels may diverge considerably over consumers.

The economy consists of $n$ consumers, each having an infinite planning horizon. Each consumer $i$ (where $i=1, \ldots, n$ ) has a utility function of the constant absolute risk aversion $(C A R A)$ type, namely $u\left(c_{i t}\right)=(-1 / \gamma) e^{-\gamma c_{i t}}$ where $c_{i t}$ is real consumption of consumer $i$ in period $t$ and where $\gamma$ is the coefficient of absolute risk aversion $(\gamma>0)$ which also equals the coefficient of absolute prudence. We use this type of utility function instead of the more usual utility function of the constant relative risk aversion $(C R R A)$ type because of its analytical convenience, i.e. it facilitates aggregation. We discuss the problem with this assumption and how to resolve it in section 5 . We further assume that all consumers can freely lend and borrow, i.e. capital markets are perfect. We assume that all consumers face the same constant real interest rate $r$ which equals their rate of time preference. ${ }^{3}$ Unlike capital markets, insurance markets are incomplete. That is, consumers cannot insure themselves through the use of so-called Arrow securities (see Deaton 1992 p.35-36) that could be traded among them to smooth consumption across different states of the world.

Given the stated assumptions, the first-order condition in period $t+1$ for consumer $i$ is,

$$
E_{i t}\left(e^{-\gamma \Delta c_{i t+1}}\right)=1
$$

\footnotetext{
${ }^{3}$ This implies that intertemporal substitution effects caused by the (anticipated) interest rate are ruled out. There is evidence that the ex ante real interest rate has no impact on consumption growth (see Hall 1988, Campbell and Mankiw 1990 or Ludvigson 1999) even when time-varying income risk is taken into account (see Parker and Preston, 2005).
} 
where $E_{i t}$ is the expectations operator conditional on information set $\Omega_{i t}$ available to consumer $i$ in period $t$. Using a second-order Taylor expansion of $e^{-\gamma \Delta c_{i t+1}}$ around $E_{i t} \Delta c_{i t+1}$ we rewrite eq.(1) as,

$$
\Delta c_{i t+1}=\frac{\gamma}{2} E_{i t} \varepsilon_{c i t+1}^{2}+\varepsilon_{c i t+1}
$$

where $\varepsilon_{c i t+1}=c_{i t+1}-E_{i t} c_{i t+1}($ see appendix A).

The period $t+1$ budget constraint under which the optimization takes place is given by,

$$
w_{i t+1}^{f}=(1+r) w_{i t}^{f}+y_{i t+1}-c_{i t+1}
$$

where the variable $w_{i t}^{f}$ is consumer $i$ 's financial wealth at the end of period $t$ and where $y_{i t+1}$ is consumer $i$ 's after-tax labour income. Following Demery and Duck (2000) we model $y_{i t+1}$, which is the exogenous process driving the model, as consisting of an aggregate component and an individual-specific component. Both components are modelled as ARIMA processes. The errors follow a GARCH process (see Meghir and Pistaferri 2004). Aggregate after-tax labour income $y_{t+1}$ is modelled as an $A R I M A(p 1,1, q 1)$ process giving,

$$
\pi(L)\left(\Delta y_{t+1}-\mu\right)=\pi^{*}(L) \varepsilon_{y t+1}
$$

where $\pi(L)$ and $\pi^{*}(L)$ are polynomials in the lag operator $L$ of respectively order $p 1$ and $q 1$, where $\mu$ is the mean and $\varepsilon_{y t+1}$ is the aggregate labour income shock which is assumed to be white noise. We assume that it follows a $G A R C H(1,1)$ process,

$$
\varepsilon_{y t+1}^{2}=\delta_{1}+\delta_{2} \varepsilon_{y t}^{2}+\delta_{3} E_{i t-1} \varepsilon_{y t}^{2}+\omega_{t+1}^{\varepsilon}
$$

where $\delta_{1}, \delta_{2}, \delta_{3}>0$ and where $\delta_{2}+\delta_{3}<1$. The term $\omega_{t+1}^{\varepsilon}=\varepsilon_{y t+1}^{2}-E_{i t} \varepsilon_{y t+1}^{2}$ is white noise (bounded from below, see Hamilton 1994). Idiosyncratic labour income is modelled as an $A R I M A(p 2,1, q 2)$ process,

$$
\phi(L)\left(\Delta y_{i t+1}-\Delta y_{t+1}\right)=\phi^{*}(L) \eta_{i t+1}
$$

where $\phi(L)$ and $\phi^{*}(L)$ are polynomials in the lag operator $L$ of respectively order $p 2$ and $q 2$ and where $\eta_{i t+1}$ is an idiosyncratic labour income shock which is assumed to be white noise. It further has a constant unconditional variance across consumers. Also, it is uncorrelated across individuals, so that it disappears on aggregation over consumers, i.e. $n^{-1} \sum_{i=1}^{n} \eta_{i t+1}=0$. The term $\eta_{i t+1}$ is assumed to follow a $\operatorname{GARCH}(1,1)$ process,

$$
\eta_{i t+1}^{2}=\xi_{1}+\xi_{2} \eta_{i t}^{2}+\xi_{3} E_{i t-1} \eta_{i t}^{2}+\omega_{i t+1}^{\eta}
$$


where $\xi_{1}, \xi_{2}, \xi_{3}>0$ and where $\xi_{2}+\xi_{3}<1$. The term $\omega_{i t+1}^{\eta}=\eta_{i t+1}^{2}-E_{i t} \eta_{i t+1}^{2}$ is white noise (bounded from below). Since it is uncorrelated across consumers we have that $n^{-1} \sum_{i=1}^{n} \omega_{i t+1}^{\eta}=0$. Note finally that the errors $\varepsilon_{y t+1}, \eta_{i t+1}, \omega_{t+1}^{\varepsilon}$ and $\omega_{i t+1}^{\eta}$ are assumed to be mutually uncorrelated.

These assumptions lead to the following consumption function,

$$
\begin{aligned}
c_{i t}= & \frac{1-\alpha}{\alpha}\left[w_{i t}^{f}+\sum_{j=1}^{\infty} \alpha^{j} E_{i t} y_{i t+j}\right] \\
& -\frac{1-\alpha}{\alpha}\left[\sum_{j=1}^{\infty} \alpha^{j} \sum_{k=1}^{j} \frac{\gamma}{2} E_{i t} \varepsilon_{c i t+k}^{2}\right]
\end{aligned}
$$

where $\alpha=(1+r)^{-1}$. For the proof we refer to appendix B. The first term equals consumption under certainty equivalence, i.e. this is the consumption level under the standard permanent income hypothesis. The second term is the contribution of precaution which decreases consumption relative to its level under certainty equivalence. It equals the sum of future discounted variances of consumption shocks conditional on period $t$ information.

We then derive an expression for the change in consumption at the individual level,

$$
\begin{aligned}
\Delta c_{i t+1}= & A \varepsilon_{y t+1}+B \eta_{i t+1}+\frac{\gamma}{2} A^{2} E_{i t} \varepsilon_{y t+1}^{2}+\frac{\gamma}{2} B^{2} E_{i t} \eta_{i t+1}^{2} \\
& -\frac{\gamma}{2} A^{2} \frac{\delta_{2}}{1-\delta_{2}-\delta_{3}} \omega_{t+1}^{\varepsilon}-\frac{\gamma}{2} B^{2} \frac{\xi_{2}}{1-\xi_{2}-\xi_{3}} \omega_{i t+1}^{\eta}
\end{aligned}
$$

where $A$ and $B$ are complicated functions of respectively the parameters of the aggregate and of the idiosyncratic labour income process. For the proof we refer to appendix C. From eq.(9) we note that the change in individual consumption from period $t$ to $t+1$ is determined by the idiosyncratic shock in labour income $\eta_{i t+1}$ and by the aggregate labour income shock $\varepsilon_{y t+1}$. Without uncertainty, i.e. under certainty equivalence, only these two shocks affect the change in consumption. Consumption changes are unpredictable under certainty equivalence (see Hall, 1978). With uncertainty, however, additional terms enter the equation. The change in consumption is then also determined by two income uncertainty terms. Aggregate labour income uncertainty is captured by the conditional variance of the aggregate labour income shocks $E_{i t} \varepsilon_{y t+1}^{2}$. Its effect on consumption depends on the degree of risk aversion $\gamma$ and on the parameters of the aggregate labour income process (captured by the parameter $A$ ). Idiosyncratic labour income uncertainty is captured by the term $E_{i t} \eta_{i t+1}^{2}$. Its effect also depends on the degree of risk aversion $\gamma$ and on the characteristics of the individual-specific part of labour income (captured by the parameter $B$ ). Both income risk terms enter the equation with a positive sign. The reason is that if in period $t$ 
the variance of the labour income shocks is expected to increase then consumption in period $t$ falls because consumers save more. The change in consumption from $t$ to $t+1$ is then higher because resources are transferred to the future, i.e. the consumption path becomes steeper. Finally, the shocks $\omega_{t+1}^{\varepsilon}$ and $\omega_{i t+1}^{\eta}$ capture the revisions in variance forecasts of both labour income shocks and enter the equation with a negative sign. Suppose for instance that $\omega_{t+1}^{\varepsilon}>0$, then the change in consumption from $t+2$ on will be higher because consumers update their expected variance $E_{i t+1} \varepsilon_{y t+2}^{2}$. To accommodate the larger slope of the consumption path without violating the budget constraint, period $t+1$ consumption must fall. The more persistent the effect of the shocks $\omega_{t+1}^{\varepsilon}$, that is the closer $\delta_{2}+\delta_{3}$ is to 1 , the longer it will take before the consumption slope returns to its original level and the stronger is the necessary adjustment in period $t+1$ consumption.

After aggregation of eq.(9) we obtain,

$$
\begin{aligned}
\Delta c_{t+1}= & A \varepsilon_{y t+1}+\frac{\gamma}{2} A^{2} E_{t} \varepsilon_{y t+1}^{2}+\frac{\gamma}{2} B^{2} E_{t} \eta_{t+1}^{2} \\
& -\frac{\gamma}{2} A^{2} \frac{\delta_{2}}{1-\delta_{2}-\delta_{3}} \omega_{t+1}^{\varepsilon}
\end{aligned}
$$

where $\Delta c_{t+1}=n^{-1} \sum_{i=1}^{n} \Delta c_{i t+1}, \eta_{t+1}^{2}=n^{-1} \sum_{i=1}^{n} \eta_{i t+1}^{2}$, and $E_{t}$ is the expectations operator conditional on the aggregate period $t$ information set $\Omega_{t}$. We refer to appendix $\mathrm{D}$ for the proof. The idiosyncratic shocks $\eta_{i t+1}$ and $\omega_{i t+1}^{\eta}$ average out but the average of the conditional variances of $\eta_{i t+1}$, i.e. idiosyncratic labour income risk in the aggregate as captured by $E_{t} \eta_{t+1}^{2}$, affects the change in aggregate consumption.

Preliminary estimations suggest that over the sample period aggregate labour income can be represented by a random walk (with drift), namely $y_{t+1}=\mu+y_{t}+\varepsilon_{y t+1}$. This implies that, in eq.(4), we have $\pi(L)=\pi^{*}(L)=1$ leading to $A=1$ so that eq.(10) now becomes,

$$
\Delta c_{t+1}=\varepsilon_{y t+1}+\frac{1}{2} \gamma E_{t} \varepsilon_{y t+1}^{2}+\frac{1}{2} \gamma B^{2} E_{t} \eta_{t+1}^{2}+\omega_{t+1}
$$

where $\omega_{t+1}=-\frac{\gamma}{2} \frac{\delta_{2}}{1-\delta_{2}-\delta_{3}} \omega_{t+1}^{\varepsilon}$. Thus, given the random walk assumption for aggregate labour income, an income shock $\varepsilon_{y t+1}$ leads to a one-for-one change in permanent income and in consumption. 


\section{Methodology.}

\subsection{Empirical specification.}

In this section we present our empirical specification. While aggregate labour income risk is modelled through a $G A R C H(1,1)$ process on aggregate labour income shocks, the contribution to aggregate consumption of idiosyncratic labour income risk is modelled as an unobserved component. We estimate the following system,

$$
\begin{gathered}
\Delta c_{t+1}=\frac{1}{2} \gamma h_{t+1}+\psi_{t+1}+\varepsilon_{y t+1}+\bar{\varepsilon}_{c t+1} \\
\Delta y_{t+1}=\mu+\varepsilon_{y t+1} \\
h_{t+1}=\delta_{1}+\delta_{2} \varepsilon_{y t}^{2}+\delta_{3} h_{t} \\
\psi_{t+1}=\lambda_{1}+\lambda_{2} \psi_{t}+\lambda_{3} x_{t}+\varepsilon_{\psi t+1} \\
\bar{\varepsilon}_{c t+1}=\varepsilon_{c t+1}+\theta \varepsilon_{c t}
\end{gathered}
$$

From the consumption equation given in eq.(12) we note, first, that the change in aggregate consumption depends positively on aggregate income risk $h_{t+1} \equiv E_{t} \varepsilon_{y t+1}^{2}$. As can be seen in eq.(14) this is modelled as a $G A R C H(1,1)$ process for labour income shocks. ${ }^{4}$ Second, the change in aggregate consumption also depends on an unobserved component $\psi_{t+1}$ which encompasses consumer-specific labour income uncertainty $E_{t} \eta_{t+1}^{2}$, i.e. $\psi_{t+1} \equiv \frac{1}{2} \gamma B^{2} E_{t} \eta_{t+1}^{2}$. As can be seen in eq.(15) the unobserved component $\psi_{t+1}$ is assumed to follow an $A R(1)$ process with a predetermined variable vector $x_{t}$. Third, given the random walk assumption for the aggregate labour income process given in eq.(13), the theoretical model derived in section 2 predicts that every shock in labour income is permanent and leads to a one for one change in consumption. Therefore the error term $\varepsilon_{y t+1}$ enters the consumption equation with coefficient equal to 1 (see also eq.11). Fourth, as far as the error term $\bar{\varepsilon}_{c t+1}$ is concerned, we note that it contains revisions in income variance forecasts $\omega_{t+1}$ (see eq.11) but that it may also contain transitory consumption and measurement error (see Deaton 1992). Suppose that we denote a transitory component and/or measurement error in the level of consumption by the white noise term $\xi_{t+1}$. Then $\bar{\varepsilon}_{c t+1}=\omega_{t+1}+\xi_{t+1}-\xi_{t}$. We

\footnotetext{
${ }^{4}$ This specification follows in a straightforward fashion from eq.(5) in the theoretical model. To see this note that a $\operatorname{GARCH}(1,1)$ model can be written as an $A R C H(\infty)$ model. Eq.(5) can be written as $\varepsilon_{y t+1}^{2}=\delta_{1}\left(1-\delta_{3}\right)^{-1}+$ $\delta_{2}\left(1-\delta_{3} L\right)^{-1} \varepsilon_{y t}^{2}+\omega_{t+1}^{\varepsilon}$ where $L$ is the lag operator. From this we note that $E_{i t} \varepsilon_{y t+1}^{2}=E_{t} \varepsilon_{y t+1}^{2}$ given that for the aggregate information set $\Omega_{t}$ and for the idiosyncratic information sets $\Omega_{i t}$ we have $\Omega_{t} \subset \Omega_{i t}(\forall i)$.
} 
can write the expression for $\bar{\varepsilon}_{c t+1}$ as in eq.(16) where $\varepsilon_{c t+1}$ is white noise and where $\theta$ depends on the relative variances of $\omega_{t+1}$ and $\xi_{t+1}$.

To identify the unobserved component $\psi_{t+1}$ as idiosyncratic labour income risk we impose two conditions. First, we impose $\sigma_{\psi}^{2}=0$ (statistical identification). This restriction is an implication of the model since if $\psi_{t+1}$ reflects idiosyncratic labour risk it contains only an anticipated term. Below we discuss how to implement this restriction in a Bayesian framework. Second, to economically interpret $\psi_{t+1}$ as idiosyncratic labour income risk (economic identification) we include in $x_{t}$ two determinants of income risk suggested in the literature: the change in the unemployment rate $\Delta u_{t}$ (see e.g. Carroll 1992) and the change in the trend of the personal transfers to GDP ratio $\Delta t_{t}$ (see Hubbard et al. 1995, Engen and Gruber 2001, and Pozzi 2007), i.e. $x_{t}=\left[\begin{array}{ll}\Delta t_{t} & \Delta u_{t}\end{array}\right]^{\prime}$ with corresponding parameter vector $\lambda_{3}=\left[\begin{array}{ll}\lambda_{3}^{t} & \lambda_{3}^{u}\end{array}\right]$. We use the trend change in the transfer rate to reduce the effect of the cyclical component of transfers since this component is strongly correlated with the unemployment rate. We check whether our specification for $\psi_{t+1}$ is adequate through the application of autocorrelation tests.

In appendix $\mathrm{E}$ we report the state space representation of the system in eqs.(12)-(16). We also discuss how to tackle GARCH errors in state space models. When reporting our results we present graphs of the estimated unobserved component series $\psi_{t+1}$ and of the estimated GARCH series $h_{t+1}$. Both are obtained by application of the Kalman filter.

\subsection{Parameter estimation.}

As noted by Harvey et al. (1992) in a state space model with GARCH errors the Kalman filter can be used to construct an approximate likelihood function. We use a Bayesian approach to parameter estimation by combining this likelihood with prior parameter information. By maximizing the sum of the sample log likelihood and the log of the prior parameter distributions we obtain the mode of the posterior parameter distribution. The mode and the corresponding Hessian-based parameter covariance matrix form the basis of the importance sampling approach which is used to obtain (means, variances and percentiles of) posterior parameter distributions. We refer to appendix G for a formal exposition of these issues.

As far as the priors are concerned we impose priors on the variance of the error term of the unobserved component $\sigma_{\psi}^{2}$, on the coefficient of absolute risk aversion $\gamma$, and on the GARCH 
parameters $\delta_{2}$ and $\delta_{3}$. For the remaining parameters we have no useful prior knowledge so we impose diffuse priors.

To implement the restriction $\sigma_{\psi}^{2}=0$ we impose a degenerate prior on $\sigma_{\psi}^{2}$, i.e. a mean and a variance of 0 .

A plausible range of values for the coefficient of relative risk aversion is $(0.5,10)$. Given the variation of the variable $c_{t}$ over the sample period (see table 1) a plausible prior for the coefficient of absolute risk aversion $\gamma(>0)$ is given by a gamma distribution with mean .0003 and standard error .0001.

We also use priors for the parameters $\delta_{2}$ and $\delta_{3}$ because the estimation of a GARCH model for labour income shocks may be affected by the presence of outliers in the series for labour income changes. These outliers considerably affect the tails (kurtosis) of the distribution of this series (see figure 1 and table 1). The quadratic form of the GARCH specification tends to magnify outliers. With priors we can decrease the weight of the most recent shock $\varepsilon_{y t}^{2}$ (i.e. impose a "low" prior on $\delta_{2}$ in the estimations) and increase the weight of $h_{t}$ (i.e. impose a "high" prior on $\delta_{3}$ in the estimations). We therefore proceed as follows. We estimate the state space system with a prior for $\delta_{2}$ with mean 0.5 (and standard deviation 0.2 ) and a prior for $\delta_{3}$ with mean 0.1 (and standard deviation 0.05). ${ }^{5}$ Note that since $0<\delta_{2}, \delta_{3}<1$ we use beta distributions as prior distributions. We then check the robustness of our results if we reduce the weight of $\varepsilon_{y t}^{2}$ in $h_{t+1}$ by imposing a prior for $\delta_{2}$ with mean 0.1 (and standard deviation 0.05) and a prior for $\delta_{3}$ with mean 0.5 (and standard deviation 0.2$).{ }^{6}$

\section{Results.}

In tables 2 and 3 the estimation results are presented for the system eqs.(12)-(16). In table 2 we present the results for the GARCH priors $\left(\delta_{2}, \delta_{3}\right)=(0.5,0.1)$, while in table 3 we present the results for the priors $\left(\delta_{2}, \delta_{3}\right)=(0.1,0.5)$. From both tables we note that the modes, means, and medians of the posterior distributions of most parameters are of equal magnitude. This is an indication that the posterior distributions are symmetric. Note also that the Ljung Box test statistics for autocorrelation (see Durbin and Koopman 2001, p.153) do not reject the null hypothesis of no

\footnotetext{
${ }^{5}$ Prior estimation of eqs.(13)-(14) separately by maximum likelihood gives a significant estimate for $\delta_{2}$ of about 0.5 and a value for $\delta_{3}$ of almost 0 .

${ }^{6}$ Prior estimation of eqs.(13)-(14) with two outlier dummies in eq.(13) gives an estimate for $\delta_{2}$ near 0 and an estimate for $\delta_{3}$ of about 0.4 .
} 
autocorrelation in the one-step ahead prediction errors of both observation equations in the state space system (consumption and income). These tests therefore provide support for our empirical specification.

Based on the theoretical model, the unobserved component $\psi_{t}$ is expected to reflect idiosyncratic labour income uncertainty at the aggregate level. The interpretation of this component as reflecting idiosyncratic labour income risk is supported by the finding that it is affected by received consumer transfers. From the estimates for $\lambda_{3}^{t}$ we note that the change in the trend of the personal transfers to GDP ratio has an important negative effect on the change in consumption, e.g. from the estimates in table 2 it can be calculated that if $\Delta t_{t}$ rises with $25 \%$ of its average value then $\Delta c_{t+1}$ decreases with almost $5 \%$ of its average value (see table 1 for descriptive statistics of all variables). Thus, idiosyncratic labour income risk matters for the aggregate economy (see also Pozzi 2007). The mean of the posterior distribution of $\lambda_{3}^{u}$, the coefficient on the unemployment change, is positive - which is in accordance with what we expect on a theoretical basis - but its standard error is rather large so that zero values are present between the percentiles 5 and 95 of the distribution. The small effect of the change in the unemployment rate contradicts Storesletten et al. (2004) who use both panel and macro data to calculate idiosyncratic labour income risk and find evidence that it is countercyclical, i.e. higher in recessions. This result is also reported in Parker and Preston (2005). Primiceri and van Rens (2004), on the other hand, find no evidence of the cyclical behaviour of income risk.

In figure 3 we present the estimated unobserved state $\psi_{t}$ with $90 \%$ confidence bands. The unobserved component largely follows the change in the trend of the personal transfers to GDP ratio. In figure 4 this component is compared to the trend in the change of consumption. Both trends move together rather closely from the eighties onward suggesting that the trend in the transfers received by consumers is a good candidate to explain low frequency movements in the change in aggregate consumption in the US in the second part of the sample.

If we look at the $G A R C H$ part of the system we note from table 2 that the posterior means of the parameters $\delta_{2}$ and $\delta_{3}$ are close to the prior means while the posterior standard errors are smaller. The data puts much weight on the ARCH term. To control whether this is due only to the outliers in labour income we also estimate the system with a high prior for $\delta_{3}$ (table 3 ). From the estimated conditional variance series presented in figure 2 we note that the peaks are flattened considerably in the case presented in table 3 where priors are used to shift the weight from the ARCH term to the GARCH term. From a comparison of tables 2 and 3 we note however 
that that this has little effect on the parameters other than $\delta_{1}, \delta_{2}$, and $\delta_{3}$. The reason for this is that the variation in the conditional variance series is insufficient to explain much of the variation in consumption changes given the estimated values of the risk aversion parameter $\gamma$. Aggregate labour income risk explains not even $1 \%$ of the variance of changes in aggregate consumption. Also, given the magnitude of the estimates for $\gamma$, the average conditional variance of aggregate labour income is much too small to be in accordance with the average change in consumption over the sample period. While disappointing these results are entirely in line with the general presumption that the volatility in aggregate consumption or labour income growth is not high enough to cause consumption growth under plausible values for risk aversion (see Deaton, 1992 and Gourinchas and Parker, 2001).

In the next section we investigate whether these conclusions change when we extend our model to allow for an effect of (anticipated) income changes on consumption changes. While there we discuss the excess sensitivity of consumption to current income, in the remainder of this section we discuss excess smoothness (see Deaton 1992 for an extensive discussion of both puzzles). From table 1 it is clear that changes in consumption are less volatile than changes in labour income. Yet the finding that labour income is well described by a random walk process suggests that consumption should respond fully to every income shock (i.e. our model suggests that consumption changes one-for-one in response to shocks in labour income). This implies that, in theory, consumption changes should be at least as volatile as income changes. As this is not the case, the variances of both sides of eq.(12) can only be reconciled if there is negative correlation between some of the variables included as regressors in this equation. We find that there is in fact a significant negative correlation (unreported) between the estimated states $\varepsilon_{c t+1}$ and $\varepsilon_{y t+1}$. Since $\varepsilon_{c t+1}$ contains the period $t+1$ shock in the variance of the aggregate labour income shock (i.e. $\omega_{t+1}^{\varepsilon}$ ) and since this shock enters the consumption equation with a negative sign, a positive correlation between this variance shock and $\varepsilon_{y t+1}$ could result in the finding of a negative correlation between $\varepsilon_{c t+1}$ and $\varepsilon_{y t+1}$. We can therefore interpret the finding of negative correlation between the estimated states $\varepsilon_{c t+1}$ and $\varepsilon_{y t+1}$ as empirical support for Caballero's theoretical claim that excess smoothness is explainable when income shocks and income variance shocks are positively correlated. 


\section{Extension: rule-of-thumb consumption.}

\subsection{Extended specification.}

There are reasons why consumers may not behave according to the model derived in section 2 . In this section we alter the model derived in section 2 by allowing some consumers to consume their disposable income in each period. This gives rise to excess sensitivity of private consumption to (anticipated) disposable income. Liquidity constraints (see Campbell and Mankiw 1990) or myopia (see Flavin 1985) are often cited as reasons why consumers may choose to follow such a rule-of-thumb.

In this paper however it is the precaution-based explanation for excess sensitivity (see Carroll 1992) that is particularly relevant given our assumption of $C A R A$ preferences. Remember that to obtain the analytical results in section 2, like Caballero (1990), we assume that utility is of the $C A R A$ type. In Caballero's paper this type of utility is necessary to obtain a closed form solution for the level of consumption. Since, in this paper, we are mainly interested in consumption changes (that is, in the Euler equation) the use of $C A R A$ utility cannot be justified along these lines. However, it is easy to show that $C A R A$ utility is necessary to make aggregation across consumers possible. Under $C A R A$ utility consumption changes at the individual level are linear in the conditional variance of income shocks. Under $C R R A$ utility, on the other hand, individual consumption growth is non-linear in the conditional variance of income shocks (see e.g. Banks et al. 2001). More specifically, under $C R R A$ utility the impact of the conditional variance of income shocks on individual consumption growth varies inversely with the individual-specific wealth level. This multiplicative structure makes aggregation difficult. Avoiding these problems by using $C A R A$ utility instead of $C R R A$ utility comes at a price however. The fact that under $C A R A$ utility the wealth level does not enter the Euler equation contradicts Deaton's (1991) and Carroll's (1992) model of "buffer-stock" savers. In Carroll's model (which uses $C R R A$ preferences) consumption growth is faster for households with low wealth (all other things equal) because they are building up a buffer against income shocks. An important implication of Carroll's model is that this mechanism gives a precaution-based explanation for the observed excess sensitivity of consumption to (anticipated) income. ${ }^{7}$ He argues that when wealth is left out of the Euler equation the finding that (predicted) income growth positively affects consumption growth can be explained by noting

\footnotetext{
${ }^{7}$ Note however that there is no evidence yet that the "buffer stock" model can by itself explain the magnitude of the observed excess sensitivity of aggregate consumption to income (see Ludvigson and Michaelides 2001)
} 
that low-wealth periods may coincide with rapid income growth periods (e.g. the periods of fastest income growth might be the early stages of a recovery when wealth is low because buffer stocks have been depleted during the downturn). The implication for our results is then that by using $C A R A$ utility wealth is omitted from the Euler equation but that this omitted variable problem can be resolved by incorporating disposable income changes in the model.

To allow for an effect of current disposable income changes on the change in consumption we consider the following expression for aggregate consumption changes,

$$
\Delta c_{t+1}=\rho \Delta y_{t+1}^{d}+(1-\rho)\left[\frac{1}{2} \gamma E_{t} \varepsilon_{y t+1}^{2}+\frac{1}{2} \gamma B^{2} E_{t} \eta_{t+1}^{2}+\varepsilon_{y t+1}+\omega_{t+1}\right]
$$

where $y_{t+1}^{d}$ is aggregate disposable income and where $0 \leq \rho \leq 1$. This equation reduces to eq.(11) if $\rho=0$. Consistent with the model of section 2 the variable $y_{t+1}^{d}$ can be written as the sum of aggregate labour income and aggregate capital income in the economy (i.e aggregate disposable income),

$$
y_{t+1}^{d}=y_{t+1}+r w_{t}^{f}
$$

where $w_{t}^{f}=n^{-1} \sum_{i=1}^{n} w_{i t}^{f}$ and where $n$ is the total number of consumers in the economy. From this and given the random walk assumption for $y_{t+1}$, note that we can write $\Delta y_{t+1}^{d}=$ $E_{t} \Delta y_{t+1}^{d}+\varepsilon_{y t+1}$. Therefore we have,

$$
\Delta c_{t+1}=\rho E_{t} \Delta y_{t+1}^{d}+\varepsilon_{y t+1}+(1-\rho)\left[\frac{1}{2} \gamma E_{t} \varepsilon_{y t+1}^{2}+\frac{1}{2} \gamma B^{2} E_{t} \eta_{t+1}^{2}+\omega_{t+1}\right]
$$

Empirically, eqs.(13), (14), (15) and (16) do not change while eq.(12) is replaced by,

$$
\Delta c_{t+1}=\frac{1}{2} \gamma(1-\rho) h_{t+1}+\rho E_{t} \Delta y_{t+1}^{d}+\psi_{t+1}+\varepsilon_{y t+1}+\varepsilon_{c t+1}
$$

where $h_{t+1} \equiv E_{t} \varepsilon_{y t+1}^{2}$, and $\psi_{t+1} \equiv \frac{1}{2} \gamma(1-\rho) B^{2} E_{t} \eta_{t+1}^{2}$. The variable $E_{t} \Delta y_{t+1}^{d}$ is obtained as the fitted value from a preliminary regression of per capita disposable income changes on a number of variables that are suggested by Campbell and Mankiw (1990). We refer to appendix $\mathrm{F}$ for details. As noted in appendix $\mathrm{E}$ the changes to the state space system are minimal. There is one additional parameter to be estimated, namely $\rho$. A Bayesian prior for $\rho$ is obtained from Campbell and Mankiw (1990, table 2 row 9). The mean of $\rho$ is 0.41 with standard error 0.09 . The prior distribution is assumed to be a beta distribution. 


\subsection{Results.}

In tables 4 and 5 the results are presented for the estimation of eqs.(20) and (13)-(16) for different priors for $\delta_{2}$ and $\delta_{3}$. The conclusions drawn for the basic model are valid for the extended model as well. Structural increases in the transfer to GDP rate decrease the slope of the consumption path. Based on the model, the channel through which this occurs is through a reduction in idiosyncratic labour income risk. Again, idiosyncratic labour income uncertainty matters for the aggregate.

The higher frequency movements in the change of consumption are mostly explained by the income shock and now also by the anticipated changes in disposable income. Indeed, note that for the latter regressor the posterior mean of $\rho$ is positive with a value of 0.2 . This value however is lower than what is usually found for this excess sensitivity parameter in the literature. There are a number of reasons that can explain why the posterior mean is only half the prior mean. First, the sample period we consider is longer than the one considered by Campbell and Mankiw (1990) which is 1947-1985 - since it also contains the second half of the eighties and the nineties. During this period further financial liberalization may have reduced the number of liquidity constrained consumers leading to lower excess sensitivity (see e.g. Bacchetta and Gerlach 1997). Peersman and Pozzi (2007) find that the excess sensitivity of private consumption growth to anticipated disposable income growth is about 0.28 in the US over the period 1965-2000. Second, and more importantly in the context of the paper, most studies estimate the excess sensitivity parameter whilst improperly omitting labour income uncertainty terms. As noted also by Hahm and Steigerwald (1999) this may produce an upward bias in the excess sensitivity parameter. We take this into account by proxying idiosyncratic labour income risk with transfers and this can explain the lower value found for the excess sensitivity parameter in our estimations. Hahm and Steigerwald use survey responses to proxy income uncertainty and also find, for the US over the period 1981-1994, excess sensitivity estimates of about 0.2 .

\section{Conclusions.}

In the theoretical section of this paper the effect of labour income risk on consumption changes is decomposed into an aggregate and into an idiosyncratic part. Analytical results are obtained under $C A R A$ preferences, general $A R I M A$ processes for labour income, and $G A R C H(1,1)$ processes for labour income shocks.

Three important conclusions can be withheld from the estimation results reported in the paper. 
First, idiosyncratic labour income risk - which in this paper is proxied through aggregate transfers received by consumers - has a significant impact on aggregate consumption changes and aggregate savings. From the eighties onward the trend change in transfers received by consumers provides a reasonable explanation for the low frequency movements in US consumption changes. Second, the results confirm the general presumption that aggregate labour income risk is not volatile enough to have a significant impact on consumption growth given realistic estimates for the coefficient of risk aversion. Aggregate labour income risk does not affect aggregate consumption changes and aggregate savings. Third, the estimation results can be reconciled with the well-known excess sensitivity and excess smoothness puzzles present in US data.

\section{References}

Attanasio, O. (1999): "Consumption," in Handbook of Macroeconomics, ed. by J. Taylor, and M. Woodford, vol. 1, chap. 11. Elsevier Science.

Bacchetta, P., and S. Gerlach (1997): "Consumption and Credit Constraints: international Evidence," Journal of Monetary Economics, 40, 207-38.

Banks, J., R. Blundell, and A. Brugiavini (2001): "Risk pooling, Precautionary saving and Consumption growth," Review of Economic Studies, 68, 757-779.

Bauwens, L., M. Lubrano, and J. Richard (1999): Bayesian inference in dynamic econometric models. Oxford University Press.

Caballero, R. (1990): "Consumption puzzles and Precautionary savings," Journal of Monetary Economics, 25, 113-36.

Campbell, J., And N. Mankiw (1990): "Permanent Income, Current Income and Consumption," Journal of Business and Economic Statistics, 8, 265-79.

CARroll, C. (1992): "The buffer-stock theory of saving: some macroeconomic evidence," Brookings Papers on Economic Activity, 2, 61-156.

Deaton, A. (1991): "Savings and liquidity constraints," Econometrica, 59, 1,221-48.

- (1992): Understanding Consumption. Oxford University Press.

Demery, D., and N. Duck (2000): "Incomplete Information and the time series behaviour of Consumption," Journal of Applied Econometrics, 15, 355-366. 
Durbin, J., And S. Koopman (2001): Time series analysis by state space methods, Oxford Statistical Science Series. Oxford University Press.

Engen, E., ANd J. Gruber (2001): "Unemployment insurance and precautionary saving," Journal of Monetary Economics, 47, 545-579.

Flavin, M. (1985): "Excess Sensitivity of Consumption to Current Income: liquidity Constraints or Myopia?," Canadian Journal of Economics, 38, 117-36.

Franses, P., and D. van DiJk (1999): "Outlier detection in the GARCH(1,1) model," Erasmus University Rotterdam Econometric Institute Research Report EI-99/26.

Gourinchas, P., And J. Parker (2001): "The empirical importance of precautionary saving," American Economic Review, 91(2), 406-12.

Hahm, J., and D. Steigerwald (1999): "Consumption adjustment under time-varying income uncertainty," The Review of Economics and Statistics, 81(1), 32-40.

HALL, R. (1978): "Stochastic Implications of the Life Cycle-Permanent Income Hypothesis: theory and Evidence," Journal of Political Economy, 86(6), 971-87.

(1988): "Intertemporal Substitution in Consumption," Journal of Political Economy, 96.

Hamilton, J. (1994): Time series analysis. Princeton University Press.

Harvey, A., E. Ruiz, and E. Sentana (1992): "Unobserved component time series models with ARCH disturbances," Journal of Econometrics, 52, 129-157.

Hubbard, R. G., J. Skinner, and S. Zeldes (1995): "Precautionary saving and social insurance," Journal of Political Economy, 103, 360-399.

Kim, C., and C. Nelson (1999): State-space models with regime switching: classical and Gibbssampling approaches with applications. MIT Press.

Lettau, M., and S. Ludvigson (2001): "Consumption, aggregate wealth and expected stock returns," Journal of Finance, 56, 815-849.

Ludvigson, S. (1999): "Consumption and Credit: a model of time-varying liquidity constraints," Review of Economics and Statistics, 81(3), 434-47.

Ludvigson, S., And A. Michaelides (2001): "Does Buffer-Stock Saving Explain the Smoothness and Excess Sensitivity of Consumption," American Economic Review, 91(3), 631-47. 
Meghir, C., and L. Pistaferri (2004): "Income variance dynamics and heterogeneity," Econometrica, 72(1), 1-32.

Parker, J., And B. Preston (2005): "Precautionary savings and consumption fluctuations," American Economic Review, 95(4), 1119-1143.

Peersman, G., and L. Pozzi (2007): "Business cycle fluctuations and excess sensitivity of private consumption," Economica, Forthcoming.

Pozzi, L. (2007): "Variance matters! The excess sensitivity of aggregate consumption to government transfers.," mimeo.

Primiceri, G., and T. van Rens (2004): "Inequality over the business cycle: estimating income risk using micro-data on consumption," mimeo.

Storesletten, K., C. Telmer, and A. Yaron (2004): "Cyclical Dynamics in Idiosyncratic Labor Market Risk," Journal of Political Economy, 112(3), 695-717.

\section{Appendix A: derivation of eq.(2).}

We take a second-order Taylor expansion of $e^{-\gamma \Delta c_{i t+1}}$ around $E_{i t} \Delta c_{i t+1}$ which gives the result (after taking expectations),

$$
E_{i t} e^{-\gamma \Delta c_{i t+1}}=e^{-\gamma E_{i t} \Delta c_{i t+1}}\left[1+\frac{\gamma^{2}}{2} E_{i t}\left(c_{i t+1}-E_{i t} c_{i t+1}\right)^{2}\right]
$$

Substituting this into eq.(1) and then taking logs gives, after some rearrangements, eq.(2) in the text.

\section{Appendix B: derivation of eq.(8).}

The proof is based on Caballero (1990). Combining eqs.(4) and (6) we obtain,

$$
\Delta y_{i t+1}=\mu+A(L) \varepsilon_{y t+1}+B(L) \eta_{i t+1}
$$

where $A(L)$ and $B(L)$ are infinite order lag polynomials given by $A(L)=\pi^{*}(L) \pi(L)^{-1}=$ $A_{0}+A_{1} L+A_{2} L^{2}+\ldots$ with $\sum_{j=0}^{\infty}\left|A_{j}\right|<\infty$ and $B(L)=\phi^{*}(L) \phi(L)^{-1}=B_{0}+B_{1} L+B_{2} L^{2}+\ldots$ with $\sum_{j=0}^{\infty}\left|B_{j}\right|<\infty$ (see e.g. Hamilton 1994, chapter 2). 
After solving eq.(3) forward and imposing a transversality condition we write the intertemporal budget constraint as,

$$
w_{i t}^{f}=\sum_{j=1}^{\infty} \alpha^{j} c_{i t+j}-\sum_{j=1}^{\infty} \alpha^{j} y_{i t+j}
$$

where $\alpha=(1+r)^{-1}$ (see e.g. Deaton 1992, p.81). After adding and subtracting the term $\sum_{j=1}^{\infty} \alpha^{j} E_{i t} y_{i t+j}$ to the RHS of eq.(B2) we obtain,

$$
w_{i t}^{f}=\sum_{j=1}^{\infty} \alpha^{j} c_{i t+j}-\sum_{j=1}^{\infty} \alpha^{j}\left(y_{i t+j}-E_{i t} y_{i t+j}\right)-\sum_{j=1}^{\infty} \alpha^{j} E_{i t} y_{i t+j}
$$

With the use of eq.(B1) it is straightforward to show that,

$$
y_{i t+j}-E_{i t} y_{i t+j}=\sum_{k=1}^{j} A_{j-k}^{*} \varepsilon_{y t+k}+\sum_{k=1}^{j} B_{j-k}^{*} \eta_{i t+k}
$$

with partial sums $A_{0}^{*}=A_{0}, A_{1}^{*}=A_{0}+A_{1}, \ldots, A_{j-1}^{*}=A_{0}+A_{1}+\ldots+A_{j-1}$ and $B_{0}^{*}=B_{0}$, $B_{1}^{*}=B_{0}+B_{1}, \ldots, B_{j-1}^{*}=B_{0}+B_{1}+\ldots+B_{j-1}$.

We write eq.(2) for period $t+j$ as,

$$
c_{i t+j}=c_{i t+j-1}+\frac{1}{2} \gamma E_{i t+j-1} \varepsilon_{c i t+j}^{2}+\varepsilon_{c i t+j}
$$

Writing eq.(B5) for period $t+j-1$, substituting this into eq.(B5) and re-iterating until period $t$ gives,

$$
c_{i t+j}=c_{i t}+\sum_{k=1}^{j} \varepsilon_{c i t+k}+\sum_{k=1}^{j} \frac{\gamma}{2} E_{i t+k-1} \varepsilon_{c i t+k}^{2}
$$

By adding to and subtracting from the RHS of eq.(B6) the term $\sum_{k=1}^{j} \frac{\gamma}{2} E_{i t} \varepsilon_{c i t+k}^{2}$ we obtain,

$$
c_{i t+j}=c_{i t}+\sum_{k=1}^{j} \frac{\gamma}{2} E_{i t} \varepsilon_{c i t+k}^{2}+\sum_{k=1}^{j} \varepsilon_{c i t+k}+\sum_{k=1}^{j} \frac{\gamma}{2}\left(E_{i t+k-1} \varepsilon_{c i t+k}^{2}-E_{i t} \varepsilon_{c i t+k}^{2}\right)
$$

After substituting eqs.(B4) and (B7) into eq.(B3) we obtain,

$$
\begin{aligned}
w_{i t}^{f}= & \sum_{j=1}^{\infty} \alpha^{j}\left\{c_{i t}+\sum_{k=1}^{j} \frac{\gamma}{2}\left(E_{i t+k-1} \varepsilon_{c i t+k}^{2}-E_{i t} \varepsilon_{c i t+k}^{2}\right)\right. \\
& +\sum_{k=1}^{j} \frac{\gamma}{2} E_{i t} \varepsilon_{c i t+k}^{2}+\sum_{k=1}^{j} \varepsilon_{c i t+k} \\
& -\sum_{k=1}^{j} A_{j-k}^{*} \varepsilon_{y t+k}-\sum_{k=1}^{j} B_{j-k}^{*} \eta_{i t+k} \\
& \left.-E_{i t} y_{i t+j}\right\}
\end{aligned}
$$


Taking expectations conditional on information set $\Omega_{i t}$ of the LHS and RHS of eq.(B8) we obtain, after some rearrangements, eq.(8) in the text.

\section{Appendix C: derivation of eq.(9).}

The proof is based on Caballero (1990). Substituting eq.(8) back into eq.(B8) gives,

$$
\sum_{j=1}^{\infty} \alpha^{j}\left\{\sum_{k=1}^{j} \frac{\gamma}{2}\left(E_{i t+k-1} \varepsilon_{c i t+k}^{2}-E_{i t} \varepsilon_{c i t+k}^{2}\right)+\sum_{k=1}^{j} \varepsilon_{c i t+k}-\sum_{k=1}^{j} A_{j-k}^{*} \varepsilon_{y t+k}-\sum_{k=1}^{j} B_{j-k}^{*} \eta_{i t+k}\right\}=0
$$

The aim is now to find an expression for $\varepsilon_{c i t+k}$ in terms of the 4 shocks $\varepsilon_{y t+k}, \eta_{i t+k}, \omega_{t+k}^{\varepsilon}$ and $\omega_{i t+k}^{\eta}$ (we neglect higher-order terms, i.e. the second and higher moments of the shocks $\omega_{t+1}^{\varepsilon}$ and $\left.\omega_{i t+1}^{\eta}\right)$. We use the method of undetermined coefficients. We guess that,

$$
\varepsilon_{c i t+k}=\pi_{1} \varepsilon_{y t+k}+\pi_{2} \eta_{i t+k}+\pi_{3} \omega_{t+k}^{\varepsilon}+\pi_{4} \omega_{i t+k}^{\eta}
$$

and we find expressions for $\pi_{1}, \pi_{2}, \pi_{3}$ and $\pi_{4}$. First, using the assumption that the errors $\varepsilon_{y t+1}, \eta_{i t+1}, \omega_{t+1}^{\varepsilon}$ and $\omega_{i t+1}^{\eta}$ are mutually uncorrelated and neglecting higher-order terms (i.e. the conditional variances of $\omega_{t+1}^{\varepsilon}$ and $\left.\omega_{i t+1}^{\eta}\right)$ we find, using eq.(C2),

write $E_{i t+k-1} \varepsilon_{c i t+k}^{2}=E_{i t+k-1}\left(\pi_{1} \varepsilon_{y t+k}+\pi_{2} \eta_{i t+k}+\pi_{3} \omega_{t+k}^{\varepsilon}+\pi_{4} \omega_{i t+k}^{\eta}\right)^{2}=$

$\pi_{1}^{2} E_{i t+k-1} \varepsilon_{y t+k}^{2}+\pi_{2}^{2} E_{i t+k-1} \eta_{i t+k}^{2}$. Similarly we can write $E_{i t} \varepsilon_{c i t+k}^{2}=$

$\pi_{1}^{2} E_{i t} \varepsilon_{y t+k}^{2}+\pi_{2}^{2} E_{i t} \eta_{i t+k}^{2}$. After subtracting the second result from the first we obtain,

$$
E_{i t+k-1} \varepsilon_{c i t+k}^{2}-E_{i t} \varepsilon_{c i t+k}^{2}=\pi_{1}^{2}\left(E_{i t+k-1} \varepsilon_{y t+k}^{2}-E_{i t} \varepsilon_{y t+k}^{2}\right)+\pi_{2}^{2}\left(E_{i t+k-1} \eta_{i t+k}^{2}-E_{i t} \eta_{i t+k}^{2}\right)
$$

Second, we find expressions for $E_{i t+k-1} \varepsilon_{y t+k}^{2}-E_{i t} \varepsilon_{y t+k}^{2}$ and $E_{i t+k-1} \eta_{i t+k}^{2}-E_{i t} \eta_{i t+k}^{2}$. We only present the derivation of $E_{i t+k-1} \varepsilon_{y t+k}^{2}-E_{i t} \varepsilon_{y t+k}^{2}$ as the derivation of $E_{i t+k-1} \eta_{i t+k}^{2}-E_{i t} \eta_{i t+k}^{2}$ is completely identical. Note that we can write eq.(5) as $\varepsilon_{y t+1}^{2}=\delta_{1}+\left(\delta_{2}+\delta_{3}\right) \varepsilon_{y t}^{2}-\delta_{3} \omega_{t}^{\varepsilon}+\omega_{t+1}^{\varepsilon}$ and for period $t+k$ as $\varepsilon_{y t+k}^{2}=\delta_{1}+\left(\delta_{2}+\delta_{3}\right) \varepsilon_{y t+k-1}^{2}-\delta_{3} \omega_{t+k-1}^{\varepsilon}+\omega_{t+k}^{\varepsilon}$. After repeated backward substitution we obtain,

$$
\begin{aligned}
\varepsilon_{y t+k}^{2}= & \delta_{1}\left(1+\left(\delta_{2}+\delta_{3}\right)+\left(\delta_{2}+\delta_{3}\right)^{2}+\ldots+\left(\delta_{2}+\delta_{3}\right)^{k-1}\right) \\
& +\omega_{t+k}^{\varepsilon}+\omega_{t+k-1}^{\varepsilon}\left(\delta_{2}+\delta_{3}\right)^{0} \delta_{2}+\omega_{t+k-2}^{\varepsilon}\left(\delta_{2}+\delta_{3}\right)^{1} \delta_{2} \\
& +\omega_{t+k-3}^{\varepsilon}\left(\delta_{2}+\delta_{3}\right)^{2} \delta_{2}+\ldots+\omega_{t+1}^{\varepsilon}\left(\delta_{2}+\delta_{3}\right)^{k-2} \delta_{2} \\
& -\omega_{t}^{\varepsilon}\left(\delta_{2}+\delta_{3}\right)^{k-1} \delta_{3}+\left(\delta_{2}+\delta_{3}\right)^{k} \varepsilon_{y t}^{2}
\end{aligned}
$$


Taking expectations of eq.(C4) with respect to info set $\Omega_{i t+k-1}$ and info set $\Omega_{i t}$ and subtracting the last result from the first we obtain,

$$
E_{i t+k-1} \varepsilon_{y t+k}^{2}-E_{i t} \varepsilon_{y t+k}^{2}=\sum_{h=1}^{k-1} \delta_{2}\left(\delta_{2}+\delta_{3}\right)^{k-1-h} \omega_{t+h}^{\varepsilon}
$$

Similarly we can write,

$$
E_{i t+k-1} \eta_{i t+k}^{2}-E_{i t} \eta_{i t+k}^{2}=\sum_{h=1}^{k-1} \xi_{2}\left(\xi_{2}+\xi_{3}\right)^{k-1-h} \omega_{i t+h}^{\eta}
$$

Using eqs.(C5) and (C6) into eq.(C3) and the result into eq.(C1) we can write,

$$
\begin{gathered}
\sum_{j=1}^{\infty} \alpha^{j}\left\{(j>2) \sum_{k=2}^{j} \frac{\gamma}{2} \pi_{1}^{2} \sum_{h=1}^{k-1} \delta_{2}\left(\delta_{2}+\delta_{3}\right)^{k-1-h} \omega_{t+h}^{\varepsilon}+(j>2) \sum_{k=2}^{j} \frac{\gamma}{2} \pi_{2}^{2} \sum_{h=1}^{k-1} \xi_{2}\left(\xi_{2}+\xi_{3}\right)^{k-1-h} \omega_{i t+h}^{\eta}\right. \\
\left.+\sum_{k=1}^{j} \varepsilon_{c i t+k}-\sum_{k=1}^{j} A_{j-k}^{*} \varepsilon_{y t+k}-\sum_{k=1}^{j} B_{j-k}^{*} \eta_{i t+k}\right\}=0
\end{gathered}
$$

This condition should be satisfied period-by-period since $\varepsilon_{y t+1}, \eta_{i t+1}, \omega_{t+1}^{\varepsilon}, \omega_{i t+1}^{\eta}$ and $\varepsilon_{c i t+1}$ are white noise terms. This means that the sum of the terms in $\varepsilon_{y t+1}, \eta_{i t+1}, \omega_{t+1}^{\varepsilon}, \omega_{i t+1}^{\eta}$ and $\varepsilon_{c i t+1}$ equals 0 and so on for $t+2, \ldots$ The sum of terms containing $\omega_{t+1}^{\varepsilon}$ in eq.(C7) is

$$
\sum_{j=1}^{\infty} \alpha^{j}\left[\frac{\gamma}{2} \delta_{2} \pi_{1}^{2}\left(\left(\delta_{2}+\delta_{3}\right)^{0}+\left(\delta_{2}+\delta_{3}\right)^{1}+\left(\delta_{2}+\delta_{3}\right)^{2} \ldots\right) \omega_{t+1}^{\varepsilon}\right]
$$

or $\frac{\alpha}{1-\alpha} \frac{\gamma}{2} \pi_{1}^{2} \frac{\delta_{2}}{1-\delta_{2}-\delta_{3}} \omega_{t+1}^{\varepsilon}$.

Similarly for $\omega_{i t+1}^{\eta}$ we have $\frac{\alpha}{1-\alpha} \frac{\gamma}{2} \pi_{2}^{2} \frac{\xi_{2}}{1-\xi_{2}-\xi_{3}} \omega_{i t+1}^{\eta}$.

The sum of terms in $\varepsilon_{c i t+1}$ is given by $\sum_{j=1}^{\infty} \alpha^{j} \varepsilon_{c i t+1}=\frac{\alpha}{1-\alpha} \varepsilon_{c i t+1}$.

The terms in $\varepsilon_{y t+1}$ are given by $\sum_{j=1}^{\infty} \alpha^{j} A_{j-1}^{*} \varepsilon_{y t+1}$ where we note, from the definition of the partial sum $A_{j-1}^{*}$ in the main text, that $\sum_{j=1}^{\infty} \alpha^{j} A_{j-1}^{*}=\sum_{j=1}^{\infty} \alpha^{j}\left(A_{0}+A_{1}+\ldots .+A_{j-1}\right)$. It is easy to show that this expression can be written as $\frac{\alpha}{1-\alpha}\left(A_{0}+A_{1} \alpha+A_{2} \alpha^{2}+\ldots.\right)$ so that for the terms in $\varepsilon_{y t+1}$ we have $\frac{\alpha}{1-\alpha} \sum_{j=0}^{\infty} \alpha^{j} A_{j} \varepsilon_{y t+1}=\frac{\alpha}{1-\alpha} A \varepsilon_{y t+1}$ where $A=\sum_{j=0}^{\infty} \alpha^{j} A_{j}<\infty$ since $\sum_{j=0}^{\infty} A_{j}<\infty$.

Similarly for the terms in $\eta_{i t+1}$ we find $\frac{\alpha}{1-\alpha} \sum_{j=0}^{\infty} \alpha^{j} B_{j} \eta_{i t+1}=\frac{\alpha}{1-\alpha} B \eta_{i t+1}$ where $B=\sum_{j=0}^{\infty} \alpha^{j} B_{j}<$ $\infty$ since $\sum_{j=0}^{\infty} B_{j}<\infty$.

Adding the terms in $\varepsilon_{y t+1}, \eta_{i t+1}, \omega_{t+1}^{\varepsilon}, \omega_{i t+1}^{\eta}$ and $\varepsilon_{c i t+1}$ and setting equal to zero gives,

$$
\varepsilon_{c i t+1}=A \varepsilon_{y t+1}+B \eta_{i t+1}-\frac{\gamma}{2} A^{2} \frac{\delta_{2}}{1-\delta_{2}-\delta_{3}} \omega_{t+1}^{\varepsilon}-\frac{\gamma}{2} B^{2} \frac{\xi_{2}}{1-\xi_{2}-\xi_{3}} \omega_{i t+1}^{\eta}
$$


where $A=\sum_{j=0}^{\infty} A_{j} \alpha^{j}$ and $B=\sum_{j=0}^{\infty} B_{j} \alpha^{j}$ with $A_{j}$ and $B_{j}(\forall j)$ as defined above. ${ }^{8}$ From confronting eq.(C2) and eq.(C8) we thus find $\pi_{1}=A, \pi_{2}=B, \pi_{3}=-\frac{\gamma}{2} A^{2} \frac{\delta_{2}}{1-\delta_{2}-\delta_{3}}$ and $\pi_{4}=$ $-\frac{\gamma}{2} B^{2} \frac{\xi_{2}}{1-\xi_{2}-\xi_{3}}$. By substituting this result into eq.(2) and neglecting the higher-order terms (i.e. the conditional variances of $\omega_{t+k}^{\varepsilon}$ and $\omega_{i t+k}^{\eta}$ ) we obtain eq.(9) in the text.

\section{Appendix D: derivation of eq.(10).}

Averaging eq.(9) over the $n$ consumers gives,

$$
\begin{aligned}
\Delta c_{t+1}= & \frac{\gamma}{2} A^{2} n^{-1} \sum_{i=1}^{n} E\left[\varepsilon_{y t+1}^{2} \mid \Omega_{i t}\right] \\
& +\frac{\gamma}{2} B^{2} n^{-1} \sum_{i=1}^{n} E\left[\eta_{i t+1}^{2} \mid \Omega_{i t}\right]+A \varepsilon_{y t+1} \\
& -\frac{\gamma}{2} A^{2} \frac{\delta_{2}}{1-\delta_{2}-\delta_{3}} \omega_{t+1}^{\varepsilon}
\end{aligned}
$$

where $\Delta c_{t+1}=n^{-1} \sum_{i=1}^{n} \Delta c_{i t+1}$ and where we use $n^{-1} \sum_{i=1}^{n} \eta_{i t+1}=0$

and $n^{-1} \sum_{i=1}^{n} \omega_{i t+1}^{\eta}=0$ to obtain the result. Note that for the aggregate information set in period $t, \Omega_{t}$, we have $\Omega_{t} \subset \Omega_{i t}(\forall i)$. By taking expectations of the LHS and of the RHS of eq.(D1) conditional on the information set $\Omega_{t}$ we obtain, after using the law of iterated expectations,

$$
\begin{aligned}
E\left[\Delta c_{t+1} \mid \Omega_{t}\right]= & \frac{\gamma}{2} A^{2} n^{-1} \sum_{i=1}^{n} E\left[\varepsilon_{y t+1}^{2} \mid \Omega_{t}\right] \\
& +\frac{\gamma}{2} B^{2} n^{-1} \sum_{i=1}^{n} E\left[\eta_{i t+1}^{2} \mid \Omega_{t}\right]
\end{aligned}
$$

Note that this result follows from the fact that we assume that $\varepsilon_{y t+1}$ and $\omega_{t+1}^{\varepsilon}$ cannot be predicted with info set $\Omega_{i t}$ for $i=1, \ldots, n$. Since $\Omega_{t} \subset \Omega_{i t}(\forall i)$ these terms cannot be predicted with info set $\Omega_{t}$ either. Note further that the difference between $\Delta c_{t+1}$ and $E\left[\Delta c_{t+1} \mid \Omega_{t}\right]$ (i.e. the period $t$ "surprise" in the aggregate change in consumption) equals $A \varepsilon_{y t+1}-\frac{\gamma}{2} A^{2} \frac{\delta_{2}}{1-\delta_{2}-\delta_{3}} \omega_{t+1}^{\varepsilon}$. So, after adding $\Delta c_{t+1}-E\left[\Delta c_{t+1} \mid \Omega_{t}\right]$ to the RHS and LHS of eq.(D2) and forcing the summation signs through the expectations operators we obtain eq.(10) in the text.

\footnotetext{
${ }^{8}$ It is easy to show that $A$ and $B$ are finite. For instance, for $A$, note that given that $\sum_{j=0}^{\infty}\left|A_{j}\right|<\infty$ the theory on convergence of series implies $\lim _{j \rightarrow \infty}\left|\frac{A_{j+1}}{A_{j}}\right| \leq 1$. Since $0<\alpha<1$ this implies that $\lim _{j \rightarrow \infty}\left|\frac{\alpha A_{j+1}}{A_{j}}\right|<1$. Multiplying numerator and denominator by $\alpha^{j}$ gives $\lim _{j \rightarrow \infty}\left|\frac{A_{j+1} \alpha^{j+1}}{A_{j} \alpha^{j}}\right|<1$. This condition implies that the series $A_{0}+A_{1} \alpha+A_{2} \alpha^{2}+\ldots$. converges.
} 


\section{Appendix E: state space system.}

We write eqs.(12)-(16) as a Gaussian linear state space system with GARCH effects (see Harvey et al. (1992) and Kim and Nelson 1999, chapter 6) where the state vector is $S_{t+1}$,

$$
\begin{gathered}
m_{t+1}=Z_{t+1} S_{t+1}+\varepsilon_{t+1} \\
S_{t+1}=T_{t+1} S_{t}+\pi_{t+1}
\end{gathered}
$$

with

$$
\begin{aligned}
& \varepsilon_{t+1 \mid \Omega_{t}} \sim N\left(0, H_{t+1}\right) \\
& \pi_{t+1 \mid \Omega_{t}} \sim N\left(0, Q_{t+1}\right)
\end{aligned}
$$

where

$$
\begin{aligned}
& m_{t+1}=\left[\begin{array}{ll}
\Delta c_{t+1} & \Delta y_{t+1}
\end{array}\right]^{\prime}, S_{t+1}=\left[\begin{array}{lllll}
1 & \varepsilon_{y t+1} & \varepsilon_{c t+1} & \varepsilon_{c t} & \psi_{t+1}
\end{array}\right]^{\prime}, \varepsilon_{t+1}=\left[\begin{array}{ll}
0 & 0
\end{array}\right]^{\prime}, \\
& H_{t+1}=\left[\begin{array}{ll}
0 & 0 \\
0 & 0
\end{array}\right], Z_{t+1}=\left[\begin{array}{ccccc}
\frac{1}{2} \gamma h_{t+1} & 1 & 1 & \theta & 1 \\
\mu & 1 & 0 & 0 & 0
\end{array}\right], \pi_{t+1}=\left[\begin{array}{lllll}
0 & \varepsilon_{y t+1} & \varepsilon_{c t+1} & 0 & \varepsilon_{\psi t+1}
\end{array}\right]^{\prime}, \\
& T_{t+1}=\left[\begin{array}{ccccc}
1 & 0 & 0 & 0 & 0 \\
0 & 0 & 0 & 0 & 0 \\
0 & 0 & 0 & 0 & 0 \\
0 & 0 & 1 & 0 & 0 \\
\left(\lambda_{1}+\lambda_{3} x_{t}\right) & 0 & 0 & 0 & \lambda_{2}
\end{array}\right], Q_{t+1}=\left[\begin{array}{ccccc}
0 & 0 & 0 & 0 & 0 \\
0 & h_{t+1} & 0 & 0 & 0 \\
0 & 0 & \sigma_{c}^{2} & 0 & 0 \\
0 & 0 & 0 & 0 & 0 \\
0 & 0 & 0 & 0 & \sigma_{\psi}^{2}
\end{array}\right]
\end{aligned}
$$

and where $\sigma_{c}^{2}$ is the variance of $\varepsilon_{c t+1}$, and $\sigma_{\psi}^{2}$ is the variance of $\varepsilon_{\psi t+1} \cdot{ }^{9}$

To initialize the system we use $E_{0} S_{1}=\left[\begin{array}{c}1 \\ 0 \\ 0 \\ 0 \\ \frac{\lambda_{1}+\lambda_{3} x_{0}}{1-\lambda_{2}}\end{array}\right]$, and $V_{0} S_{1}=\left[\begin{array}{ccccc}0 & 0 & 0 & 0 & 0 \\ 0 & \frac{\delta_{1}}{1-\delta_{2}-\delta_{3}} & 0 & 0 & 0 \\ 0 & 0 & \sigma_{c}^{2} & 0 & 0 \\ 0 & 0 & 0 & \sigma_{c}^{2} & 0 \\ 0 & 0 & 0 & 0 & \frac{\sigma_{\psi}^{2}}{1-\lambda_{2}^{2}}\end{array}\right]$.

Note that in section 5 the matrix $Z_{t+1}$ is given by $Z_{t+1}=\left[\begin{array}{ccccc}\frac{1}{2} \gamma(1-\rho) h_{t+1}+\rho E_{t} \Delta y_{t+1}^{d} & 1 & 1 & \theta & 1 \\ \mu & 1 & 0 & 0 & 0\end{array}\right]$.

\footnotetext{
${ }^{9}$ Note that to apply the method proposed by Harvey et al. (1992) the conditional distributions of the errors in the state space model are assumed to be Gaussian. Given that $\varepsilon_{y t+1}$ follows a GARCH process, its unconditional distribution is of course not normal (see Hamilton 1994, p.662).
} 
The $G A R C H$ effects $h_{t+1}$ complicate the otherwise standard state space framework since $h_{t+1}$ and thus $Q_{t+1}$ is a function of the unobserved state $\varepsilon_{y t+1}$. Harvey et al. (1992) suggest to replace $h_{t+1}$ in the system by $h_{t+1}^{*}=\delta_{1}+\delta_{2} \varepsilon_{y t}^{* 2}+\delta_{3} h_{t}^{*}$ where the unobserved $\varepsilon_{y t}^{2}$ is replaced by its conditional expectation $\varepsilon_{y t}^{* 2}=E_{t} \varepsilon_{y t}^{2}$. Note that we can write $E_{t} \varepsilon_{y t}^{2}=\left(E_{t} \varepsilon_{y t}\right)^{2}+E_{t}\left[\left(\varepsilon_{y t}-E_{t} \varepsilon_{y t}\right)^{2}\right] .{ }^{10}$ From the period $t$ Kalman filter recursions we obtain $E_{t} \varepsilon_{y t}=E_{t}\left(S_{t}\right)[2,1]$ and $E_{t}\left[\left(\varepsilon_{y t}-E_{t} \varepsilon_{y t}\right)^{2}\right]=$ $V_{t}\left(S_{t}\right)[2,2]$. Thus, for given parameter values, given $h_{t}^{*}$ (which is initialized by the unconditional variance of $\left.\varepsilon_{y t+1}\right)$ and given the Kalman filter output from period $t$, namely $E_{t}\left(S_{t}\right)$ and $V_{t}\left(S_{t}\right)$, we can calculate $h_{t+1}^{*}$ and the system matrices $Q_{t+1}$ and $Z_{t+1}$. These make it possible to calculate $E_{t}\left(S_{t+1}\right), V_{t}\left(S_{t+1}\right)$ and $E_{t+1}\left(S_{t+1}\right), V_{t+1}\left(S_{t+1}\right)$, and so on....$^{11}$

\section{Appendix F: data.}

Data are quarterly and the sample period is 1952:01-2001:02 (and 1953:01-2001:02 for the estimations with fitted disposable income in section 5).

\section{Data description.}

$c_{t}$ : per capita consumption on nondurables and services excluding shoes and clothing, seasonally adjusted, at annual rates, in 1996 dollars.

$y_{t}$ : per capita after-tax labour income, seasonally adjusted, at annual rates, in 1996 dollars.

$t_{t}$ : trend obtained from Hodrick-Prescott filter applied to personal current transfer receipts (current prices, seasonally adjusted, annual rates) to gdp (current prices, seasonally adjusted, annual rates) rate in percent.

$u_{t}$ : unemployment rate in percent, seasonally adjusted.

$y_{t}^{d}$ : per capita after-tax total personal income, seasonally adjusted, at annual rates, in 1996 dollars.

$i_{t}$ : nominal 3 month T-bill rate, annual rate.

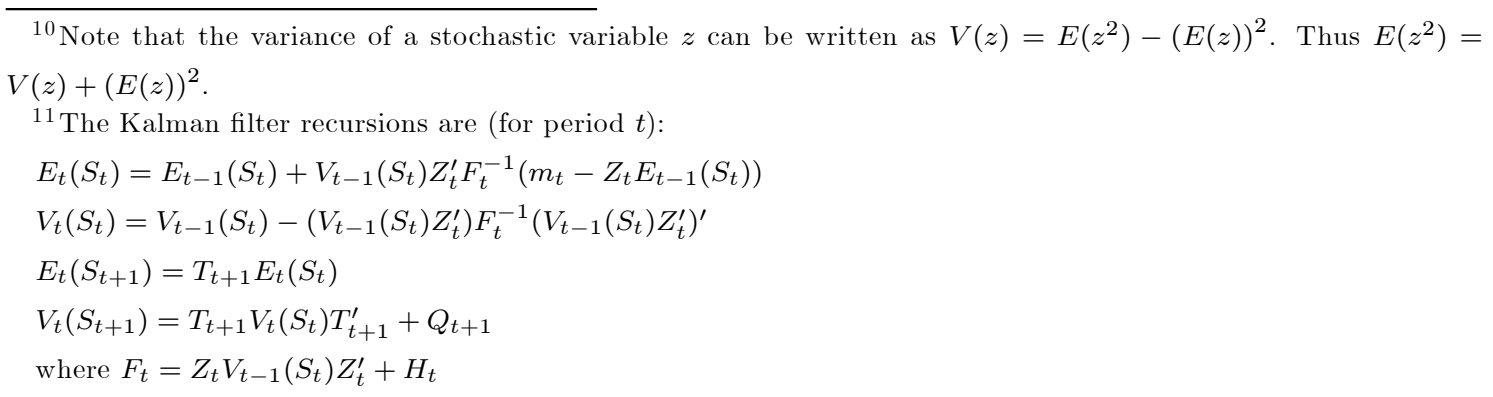


$\Delta c_{t}, \Delta y_{t}, \Delta t_{t}, \Delta u_{t}:$ first difference in $c_{t}, y_{t}, t_{t}, u_{t}$ respectively.

$E_{t-1} \Delta y_{t}^{d}$ : fitted series obtained from a least squares regression (with $\mathrm{R}^{2}=0.161$ ) of the first difference of $y_{t}^{d}$, namely $\Delta y_{t}^{d}$, on a constant, on lags 1-3 of $\Delta c_{t}$, on lags 1-3 of $\Delta y_{t}^{d}$, on lags 1-3 of the first difference of $i_{t}$, namely $\Delta i_{t}$, and on lag 1 of the error correction term $c_{t}-y_{t}^{d}$. We refer to Campbell and Mankiw (1990) for a justification of these explanatory variables for $\Delta y_{t}^{d}$.

\section{Data sources.}

$c_{t}, y_{t}$ : taken directly from updated datset for paper Lettau and Ludvigson (2001).

on http://www.econ.nyu.edu/user/ludvigsons.

$t_{t}$ : personal current transfer receipts (from table 2.1: personal income and its disposition) and gdp taken from US Department of Commerce (Bureau of Economic Analysis).

$u_{t}$ : from Bureau of Labor Statistics.

$y_{t}^{d}$ : after-tax total personal income in current prices, seasonally adjusted at annual rates, is taken from US Department of Commerce (Bureau of Economic Analysis). Deflator used is deflator for nondurables and services (minus clothing and shoes), seasonally adjusted, constructed from tables 2.3.4 and 2.3.5 US Department of Commerce (Bureau of Economic Analysis) with baseyear adjustment (from baseyear 2000 to baseyear 1996). Population is taken from Bureau of Labor Statistics.

$i_{t}$ : from IMF, International Financial Statistics.

\section{Appendix G: importance sampling.}

Suppose that $m=\left[\begin{array}{lll}m_{1}^{\prime} & \ldots & m_{T}^{\prime}\end{array}\right]^{\prime}$ (with $m_{t+1}$ as defined in appendix E) and $\Phi$ is the parameter vector. Denote the prior parameter density by $p(\Phi)$, the (sample) likelihood by $p(m \mid \Phi)$ and the posterior parameter distribution by $p(\Phi \mid m)$. Then the mode of the posterior parameter distribution is given by $\widehat{\Phi}^{o}=\arg \max [\ln p(\Phi \mid m)]=\arg \max [\ln p(\Phi)+\ln p(m \mid \Phi)]$. The corresponding Hessianbased parameter covariance matrix is obtained as $\widehat{V}^{o}=\left(\left[-\frac{\partial^{2} \ln p(\Phi)}{\partial \Phi \partial \Phi^{\prime}}-\frac{\partial^{2} \ln p(m \mid \Phi)}{\partial \Phi \partial \Phi^{\prime}}\right]_{\Phi=\widehat{\Phi}^{o}}\right)^{-1}$. We use importance sampling with sequential updating to obtain posterior parameter distributions and posterior states (see Bauwens et al 1999 chapter 3). For given $m$ the posterior state distribution is determined by knowledge of the posterior parameter distribution, so that we can restrict interest to quantities $\bar{X}$ of the form,

$$
\bar{X}=\int_{\Phi} X(\Phi) p(\Phi \mid m) d \Phi
$$


where $X(\Phi)$ is some function of the parameter vector $\Phi$. Since the posterior parameter distribution $p(\Phi \mid m)$ is unknown, we use $g(\Phi \mid m)$ as an importance density. Now we write eq.(F1) as,

$$
\bar{X}=\int_{\Phi} X(\Phi) \frac{p(\Phi \mid m)}{g(\Phi \mid m)} g(\Phi \mid m) d \Phi
$$

which, by using Bayes' law, can be rewritten as,

$$
\bar{X}=\frac{\int_{\Phi} X(\Phi) z^{g}(\Phi, m) g(\Phi \mid m) d \Phi}{\int_{\Phi} z^{g}(\Phi, m) g(\Phi \mid m) d \Phi}=\frac{E_{g}\left[X(\Phi) z^{g}(\Phi, m)\right]}{E_{g}\left[z^{g}(\Phi, m)\right]}
$$

where $E_{g}$ denotes the expectations operator with respect to $g(\Phi \mid m)$ and $z^{g}(\Phi, m)=\frac{p(\Phi) p(m \mid \Phi)}{g(\Phi \mid m)}$. We set $g(\Phi \mid m)=N\left(\widehat{\Phi}^{s}, \xi \widehat{V}^{s}\right)$ as an importance density where $\widehat{\Phi}^{s}$ and $\widehat{V}^{s}$ are sequentially updated matrices and where $\xi$ is a tuning constant (see Bauwens et al 1999). At the start of the sampling process we set $\widehat{\Phi}^{s}=\widehat{\Phi}^{o}$ and $\widehat{V}^{s}=\widehat{V}^{o}$. By taking draws $\Phi^{i}$ for $i=1, \ldots, n$ from $g(\Phi \mid m)$ we estimate $\bar{X}$ by,

$$
\widehat{X}=\frac{\sum_{i=1}^{n} X\left(\Phi^{i}\right) z^{i}}{\sum_{i=1}^{n} z^{i}}
$$

where $z^{i}=\frac{p\left(\Phi^{i}\right) p\left(m \mid \Phi^{i}\right)}{g\left(\Phi^{i} \mid m\right)}$. Parameter draws from $g(\Phi \mid m)$ that violate parameter restrictions imposed by the model are discarded. ${ }^{12}$ Posterior parameter means are calculated from eq.(F4) as $\widehat{\Phi}=\frac{\sum_{i=1}^{n} \Phi^{i} z^{i}}{\sum_{i=1}^{n} z^{i}}$. Posterior parameter covariance matrices are then calculated as $\widehat{V}(\Phi \mid m)=$ $\frac{\sum_{i=1}^{n}\left(\Phi^{i}\right)\left(\Phi^{i}\right)^{\prime} z^{i}}{\sum_{i=1}^{n} z^{i}}-\widehat{\Phi} \widehat{\Phi}^{\prime}$. We then set $\widehat{\Phi}^{s}=\widehat{\Phi}$ and $\widehat{V}^{s}=\widehat{V}$ and the sampling process is repeated. We repeat this sequential sampling process until the coefficient of variation of the weights $z^{i}$ is sufficiently reduced (see Bauwens et al. 1999, chapter 3). Further, the error bounds of the parameter means (see Bauwens et al. 1999 chapter 3, p78) indicate that the approximations of the parameter means obtained through the sampling process are of good quality (only for one parameter is the error bound somewhat high yet it is still below the "critical" threshold reported in Bauwens et al.). Note that in all cases convergence is achieved with 2, 3, or 4 updates of the importance density when setting $n=20000$ and $\xi=1.2$. The final coefficients of variation of the weights and the error bounds of the parameter means are not reported but the results are available from the author upon request. We do report the means, the variances and percentiles of the final posterior parameter distributions. Note that the $100 \mathrm{k} \%$ percentile of the posterior parameter distribution is $\Phi^{[m]}$ taken from the ordered sequence $\Phi^{[i]}$ of $\Phi^{i}$ for which $\frac{\sum_{i=1}^{m} z^{[i]}}{\sum_{i=1}^{n} z^{[i]}} \approx k$ where $z^{[i]}$ is the sequence of $z^{i}$ associated to $\Phi^{[i]}$. Note, finally, that the distributions of the posterior states (in particular, state means and state variances) are calculated by running the Kalman filter (as described in section appendix E) using the posterior parameter distributions.

\footnotetext{
${ }^{12}$ We reject draws that violate $\gamma>0, \sigma_{c}^{2}>0,-1<\lambda_{2}<1,-1<\theta<1, \delta_{1}>0, \delta_{2}>0, \delta_{3}>0$ and $\delta_{2}+\delta_{3}<1$.
} 


\section{Tables.}

Table 1: Descriptive statistics, US data, 1952:01-2001:02 (see appendix F for description and sources).

\begin{tabular}{|c|c|c|c|c|c|c|}
\hline & mean & std. dev. & maximum & minimum & skewness & kurtosis \\
\hline$c_{t}$ & 13627 & 3881.0 & 20933 & 7662.5 & 0.122 & 1.800 \\
\hline$y_{t}$ & 11235 & 3450.7 & 17374 & 5802.0 & -0.045 & 1.720 \\
\hline$t_{t}$ & 8.231 & 2.818 & 11.758 & 3.193 & -0.373 & 1.571 \\
\hline$u_{t}$ & 5.719 & 1.561 & 10.666 & 2.556 & 0.513 & 3.256 \\
\hline$\Delta c_{t}$ & 66.953 & 62.502 & 220.927 & -186.841 & -.627 & 4.379 \\
\hline$\Delta y_{t}$ & 58.489 & 96.684 & 512.242 & -410.224 & -.073 & 7.862 \\
\hline$\Delta t_{t}$ & .041 & .048 & .131 & -.038 & .209 & 2.150 \\
\hline$\Delta u_{t}$ & .005 & .380 & 1.667 & -.966 & 1.296 & 6.614 \\
\hline$E_{t-1} \Delta y_{t}^{d}$ & 74.868 & 62.656 & 240.283 & -153.493 & -.682 & 4.594 \\
\hline
\end{tabular}

Note: descriptive statistics for the series $E_{t-1} \Delta y_{t}^{d}$ are calculated over the sample period 1953:012001:02. 
Table 2: Estimation results, eqs.(12)-(16), US data, 1952:01-2001:02 ("high" prior for $\delta_{2}$ and "low" prior for $\left.\delta_{3}\right)$.

\begin{tabular}{|c|c|c|c|c|c|c|c|c|c|}
\hline coeff & \multicolumn{3}{|c|}{ prior distribution } & \multicolumn{6}{|c|}{ posterior distribution } \\
\hline & \multirow{2}{*}{ type } & \multirow{2}{*}{ mean } & \multirow[t]{2}{*}{$\operatorname{sdv}$} & \multirow[t]{2}{*}{ mode } & \multirow[t]{2}{*}{ mean } & \multirow[t]{2}{*}{$\mathrm{sdv}$} & \multicolumn{3}{|c|}{ percentiles } \\
\hline & & & & & & & 5 & 50 & 95 \\
\hline$\theta$ & diffuse & - & - & -0.3983 & -0.4161 & 0.1598 & -0.7313 & -0.3904 & -0.2046 \\
\hline$\gamma$ & gamma & .0003 & .0001 & .00027 & .00028 & .0001 & .00015 & .00027 & .00046 \\
\hline$\mu$ & diffuse & - & - & 60.137 & 59.919 & 6.298 & 49.648 & 59.937 & 70.225 \\
\hline$\delta_{1}$ & diffuse & - & - & 5221.3 & 5315.2 & 870.73 & 4019.3 & 5247.2 & 6878.0 \\
\hline$\delta_{2}$ & beta & 0.5 & 0.2 & 0.5117 & 0.5143 & 0.1436 & 0.2883 & 0.5071 & 0.7643 \\
\hline$\delta_{3}$ & beta & 0.1 & 0.05 & 0.0583 & 0.0764 & 0.0388 & 0.0245 & 0.0706 & 0.1485 \\
\hline$\lambda_{1}$ & diffuse & - & - & 115.38 & 122.05 & 23.086 & 83.162 & 122.92 & 159.06 \\
\hline$\lambda_{2}$ & diffuse & - & - & -0.5165 & -0.6198 & 0.2579 & -0.9661 & -0.6586 & -0.1381 \\
\hline$\lambda_{3}^{t}$ & diffuse & - & - & -324.92 & -336.18 & 149.78 & -587.13 & -331.79 & -99.244 \\
\hline$\lambda_{3}^{u}$ & diffuse & - & - & 8.1689 & 4.0229 & 17.491 & -24.797 & 4.1956 & 32.826 \\
\hline$\sigma_{c}^{2}$ & diffuse & - & - & 5688.3 & 5815.9 & 889.31 & 4322.9 & 5826.3 & 7243.5 \\
\hline$\sigma_{\psi}^{2}$ & degenerate & 0 & 0 & - & - & - & - & - & - \\
\hline \multicolumn{10}{|c|}{ Ljung-Box tests for autocorrelation in the one-step ahead prediction errors } \\
\hline \multicolumn{5}{|c|}{$\Delta c_{t}$} & \multicolumn{5}{|c|}{$\Delta y_{t}$} \\
\hline \multicolumn{5}{|c|}{ p-val (k) } & \multicolumn{5}{|c|}{ p-val $(\mathrm{k})$} \\
\hline \multicolumn{5}{|c|}{$0.153(4)$} & \multicolumn{5}{|c|}{0.391 (4) } \\
\hline \multicolumn{5}{|c|}{$0.373(8)$} & \multicolumn{5}{|c|}{$0.137(8)$} \\
\hline
\end{tabular}

Note: sdv denotes standard deviation. p-val $(\mathrm{k})$ denotes the p-value of the null hypothesis of no autocorrelation up to lag $\mathrm{k}$ in the one-step ahead prediction errors of the specified observation equation. 
Table 3: Estimation results, eqs.(12)-(16), US data, 1952:01-2001:02 ("low" prior for $\delta_{2}$ and "high" prior for $\left.\delta_{3}\right)$.

\begin{tabular}{|c|c|c|c|c|c|c|c|c|c|}
\hline \multirow[t]{3}{*}{ coeff } & \multicolumn{3}{|c|}{ prior distribution } & \multicolumn{6}{|c|}{ posterior distribution } \\
\hline & \multirow{2}{*}{ type } & \multirow{2}{*}{ mean } & \multirow[t]{2}{*}{$\mathrm{sdv}$} & \multirow[t]{2}{*}{ mode } & \multirow[t]{2}{*}{ mean } & \multirow[t]{2}{*}{$\mathrm{sdv}$} & \multicolumn{3}{|c|}{ percentiles } \\
\hline & & & & & & & 5 & 50 & 95 \\
\hline$\theta$ & diffuse & - & - & -0.3958 & -0.4133 & 0.1563 & -0.7283 & -0.3888 & -0.2056 \\
\hline$\gamma$ & gamma & .0003 & .0001 & .00027 & .00028 & 0.0001 & .00014 & .00027 & .00046 \\
\hline$\mu$ & diffuse & - & - & 59.827 & 59.648 & 6.693 & 48.637 & 59.567 & 70.670 \\
\hline$\delta_{1}$ & diffuse & - & - & 3898.3 & 4722.7 & 1434.6 & 2393.0 & 4691.3 & 7116.1 \\
\hline$\delta_{2}$ & beta & 0.1 & 0.05 & 0.1341 & 0.1514 & 0.0570 & 0.0689 & 0.1452 & 0.2539 \\
\hline$\delta_{3}$ & beta & 0.5 & 0.2 & 0.4330 & 0.3416 & 0.1600 & 0.0951 & 0.3324 & 0.6181 \\
\hline$\lambda_{1}$ & diffuse & - & - & 114.98 & 121.59 & 23.829 & 81.551 & 121.86 & 160.28 \\
\hline$\lambda_{2}$ & diffuse & - & - & -0.515 & -0.611 & 0.259 & -0.962 & -0.646 & -0.125 \\
\hline$\lambda_{3}^{t}$ & diffuse & - & - & -321.80 & -335.23 & 148.26 & -581.78 & -329.56 & -98.682 \\
\hline$\lambda_{3}^{u}$ & diffuse & - & - & 8.770 & 5.130 & 17.540 & -23.377 & 5.358 & 33.796 \\
\hline$\sigma_{c}^{2}$ & diffuse & - & - & 5698.2 & 5838.8 & 882.12 & 4339.1 & 5849.6 & 7270.8 \\
\hline$\sigma_{\psi}^{2}$ & degenerate & 0 & 0 & - & - & - & - & - & - \\
\hline \multicolumn{10}{|c|}{ Ljung-Box tests for autocorrelation in the one-step ahead prediction errors } \\
\hline \multicolumn{5}{|c|}{$\Delta c_{t}$} & \multicolumn{5}{|c|}{$\Delta y_{t}$} \\
\hline \multicolumn{5}{|c|}{$\mathrm{p}$-val $(\mathrm{k})$} & \multicolumn{5}{|c|}{ p-val $(\mathrm{k})$} \\
\hline \multicolumn{5}{|c|}{$0.175(4)$} & \multicolumn{5}{|c|}{$0.572(4)$} \\
\hline \multicolumn{5}{|c|}{$0.409(8)$} & \multicolumn{5}{|c|}{$0.279(8)$} \\
\hline
\end{tabular}

Note: sdv denotes standard deviation. p-val $(\mathrm{k})$ denotes the p-value of the null hypothesis of no autocorrelation up to lag $\mathrm{k}$ in the one-step ahead prediction errors of the specified observation equation. 
Table 4: Estimation results, eqs.(20) and (13)-(16), US data, 1953:01-2001:02 ("high" prior for $\delta_{2}$ and "low" prior for $\left.\delta_{3}\right)$.

\begin{tabular}{|c|c|c|c|c|c|c|c|c|c|}
\hline \multirow[t]{3}{*}{ coeff } & \multicolumn{3}{|c|}{ prior distribution } & \multicolumn{6}{|c|}{ posterior distribution } \\
\hline & \multirow{2}{*}{ type } & \multirow{2}{*}{ mean } & \multirow[t]{2}{*}{$\mathrm{sdv}$} & \multirow[t]{2}{*}{ mode } & \multirow[t]{2}{*}{ mean } & \multirow[t]{2}{*}{$\mathrm{sdv}$} & \multicolumn{3}{|c|}{ percentiles } \\
\hline & & & & & & & 5 & 50 & 95 \\
\hline$\theta$ & diffuse & - & - & -0.4306 & -0.445 & 0.1635 & -0.7713 & -0.4186 & -0.2239 \\
\hline$\gamma$ & gamma & .0003 & .0001 & .00027 & .00028 & .0001 & .00014 & .00027 & .00045 \\
\hline$\mu$ & diffuse & - & - & 59.218 & 59.519 & 6.338 & 49.173 & 59.537 & 70.011 \\
\hline$\rho$ & beta & 0.41 & 0.09 & 0.1999 & 0.2017 & 0.0488 & 0.1275 & 0.1987 & 0.2872 \\
\hline$\delta_{1}$ & diffuse & - & - & 5190.1 & 5307.1 & 887.30 & 3951.0 & 5250.2 & 6887.2 \\
\hline$\delta_{2}$ & beta & 0.5 & 0.2 & 0.5235 & 0.5208 & 0.1427 & 0.2943 & 0.5156 & 0.7654 \\
\hline$\delta_{3}$ & beta & 0.1 & 0.05 & 0.0584 & 0.0754 & 0.0384 & 0.0248 & 0.0692 & 0.1471 \\
\hline$\lambda_{1}$ & diffuse & - & - & 84.910 & 93.124 & 20.921 & 59.990 & 92.458 & 129.06 \\
\hline$\lambda_{2}$ & diffuse & - & - & -0.4362 & -0.5784 & 0.2524 & -0.9449 & -0.6023 & -0.1269 \\
\hline$\lambda_{3}^{t}$ & diffuse & - & - & -233.85 & -248.48 & 144.52 & -429.34 & -245.52 & -18.613 \\
\hline$\lambda_{3}^{u}$ & diffuse & - & - & 18.895 & 14.876 & 18.686 & -15.928 & 14.785 & 44.901 \\
\hline$\sigma_{c}^{2}$ & diffuse & - & - & 5811.5 & 6049.6 & 995.59 & 4375.1 & 6045.8 & 7705.4 \\
\hline$\sigma_{\psi}^{2}$ & degenerate & 0 & 0 & - & - & - & - & - & - \\
\hline \multicolumn{10}{|c|}{ Ljung-Box tests for autocorrelation in the one-step ahead prediction errors } \\
\hline \multicolumn{5}{|c|}{$\Delta c_{t}$} & \multicolumn{5}{|c|}{$\Delta y_{t}$} \\
\hline \multicolumn{5}{|c|}{$\mathrm{p}$-val $(\mathrm{k})$} & \multicolumn{5}{|c|}{ p-val (k) } \\
\hline \multicolumn{5}{|c|}{$0.140(4)$} & \multicolumn{5}{|c|}{$0.295(4)$} \\
\hline \multicolumn{5}{|c|}{$0.436(8)$} & \multicolumn{5}{|c|}{$0.116(8)$} \\
\hline
\end{tabular}

Note: sdv denotes standard deviation. p-val $(\mathrm{k})$ denotes the p-value of the null hypothesis of no autocorrelation up to lag $\mathrm{k}$ in the one-step ahead prediction errors of the specified observation equation. 
Table 5: Estimation results, eqs.(20) and (13)-(16), US data, 1953:01-2001:02 ("low" prior for $\delta_{2}$ and "high" prior for $\delta_{3}$ ).

\begin{tabular}{|c|c|c|c|c|c|c|c|c|c|}
\hline \multirow[t]{3}{*}{ coeff } & \multicolumn{3}{|c|}{ prior distribution } & \multicolumn{6}{|c|}{ posterior distribution } \\
\hline & \multirow[t]{2}{*}{ type } & \multirow[t]{2}{*}{ mean } & \multirow[t]{2}{*}{$\mathrm{sdv}$} & \multirow[t]{2}{*}{ mode } & \multirow[t]{2}{*}{ mean } & \multirow[t]{2}{*}{$\operatorname{sdv}$} & \multicolumn{3}{|c|}{ percentiles } \\
\hline & & & & & & & 5 & 50 & 95 \\
\hline$\theta$ & diffuse & - & - & -0.4284 & -0.4465 & 0.1658 & -0.7836 & -0.4182 & -0.2254 \\
\hline$\gamma$ & gamma & .0003 & .0001 & .00027 & .00028 & .0001 & .00014 & .00027 & .00046 \\
\hline$\mu$ & diffuse & - & - & 59.032 & 59.613 & 6.796 & 48.464 & 59.600 & 70.724 \\
\hline$\rho$ & beta & 0.41 & 0.09 & 0.1999 & 0.2019 & 0.0483 & 0.1255 & 0.1995 & 0.2842 \\
\hline$\delta_{1}$ & diffuse & - & - & 3925.2 & 4737.0 & 1465.5 & 2342.6 & 4715.4 & 7175.6 \\
\hline$\delta_{2}$ & beta & 0.1 & 0.05 & 0.1346 & 0.1513 & 0.0555 & 0.0672 & 0.1468 & 0.2514 \\
\hline$\delta_{3}$ & beta & 0.5 & 0.2 & 0.4327 & 0.3418 & 0.1594 & 0.0971 & 0.3294 & 0.6182 \\
\hline$\lambda_{1}$ & diffuse & - & - & 84.678 & 92.943 & 21.418 & 59.252 & 92.448 & 128.92 \\
\hline$\lambda_{2}$ & diffuse & - & - & -0.4346 & -0.5699 & 0.2538 & -0.9430 & -0.5941 & -0.1152 \\
\hline$\lambda_{3}^{t}$ & diffuse & - & - & -231.32 & -240.11 & 143.20 & -479.20 & -237.06 & -9.549 \\
\hline$\lambda_{3}^{u}$ & diffuse & - & - & 19.355 & 15.875 & 18.551 & -15.023 & 16.183 & 45.776 \\
\hline$\sigma_{c}^{2}$ & diffuse & - & - & 5820.2 & 6012.8 & 980.10 & 4340.6 & 6009.9 & 7630.5 \\
\hline$\sigma_{\psi}^{2}$ & degenerate & 0 & 0 & - & - & - & - & - & - \\
\hline \multicolumn{10}{|c|}{ Ljung-Box tests for autocorrelation in the one-step ahead prediction errors } \\
\hline \multicolumn{5}{|c|}{$\Delta c_{t}$} & \multicolumn{5}{|c|}{$\Delta y_{t}$} \\
\hline \multicolumn{5}{|c|}{$\mathrm{p}$-val (k) } & \multicolumn{5}{|c|}{$\mathrm{p}$-val $(\mathrm{k})$} \\
\hline \multicolumn{5}{|c|}{$0.157(4)$} & \multicolumn{5}{|c|}{$0.486(4)$} \\
\hline \multicolumn{5}{|c|}{$0.470(8)$} & \multicolumn{5}{|c|}{$0.264(8)$} \\
\hline
\end{tabular}

Note: sdv denotes standard deviation. p-val $(\mathrm{k})$ denotes the p-value of the null hypothesis of no autocorrelation up to lag $\mathrm{k}$ in the one-step ahead prediction errors of the specified observation equation.

\section{Figures.}


Figure 1: First difference of real per capita after-tax labour income, US, 1952:01-2001:02 (source: see appendix F)

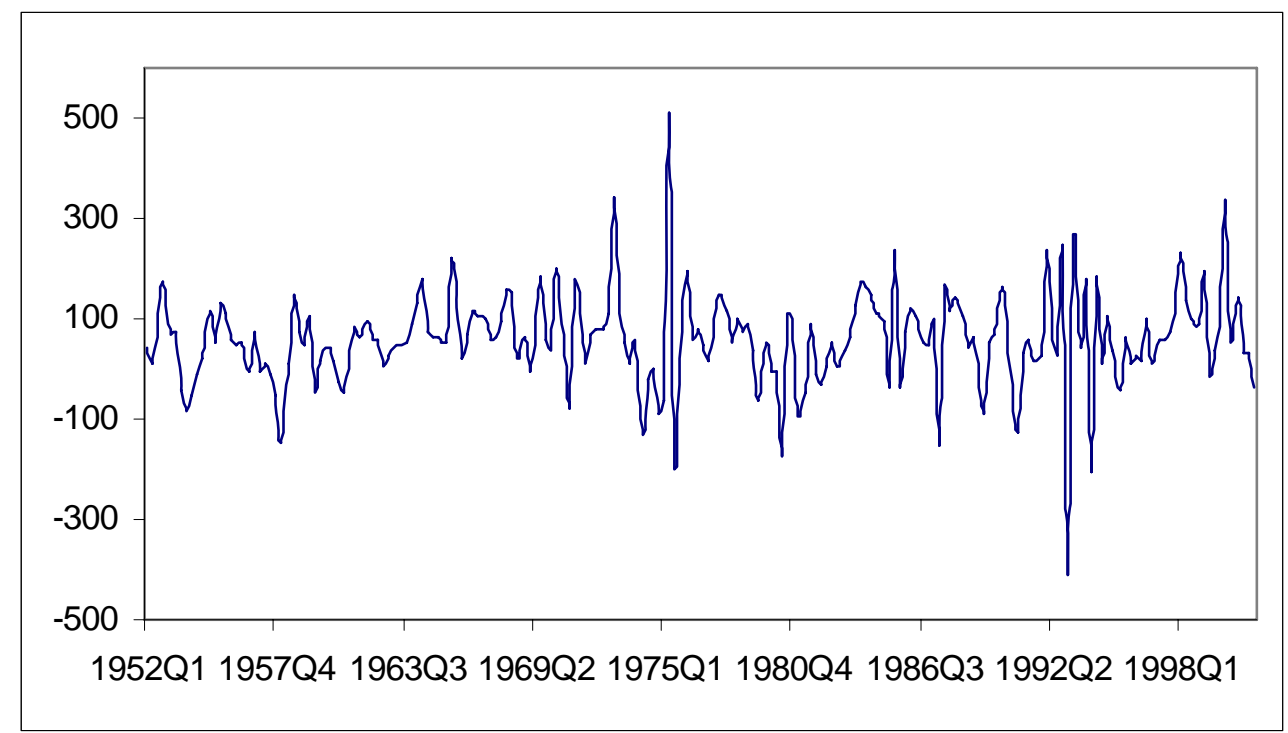

Figure 2: GARCH series $h_{t}$ with different priors on $\delta_{2}$ and $\delta_{3}$ (case tables 2 and 3)

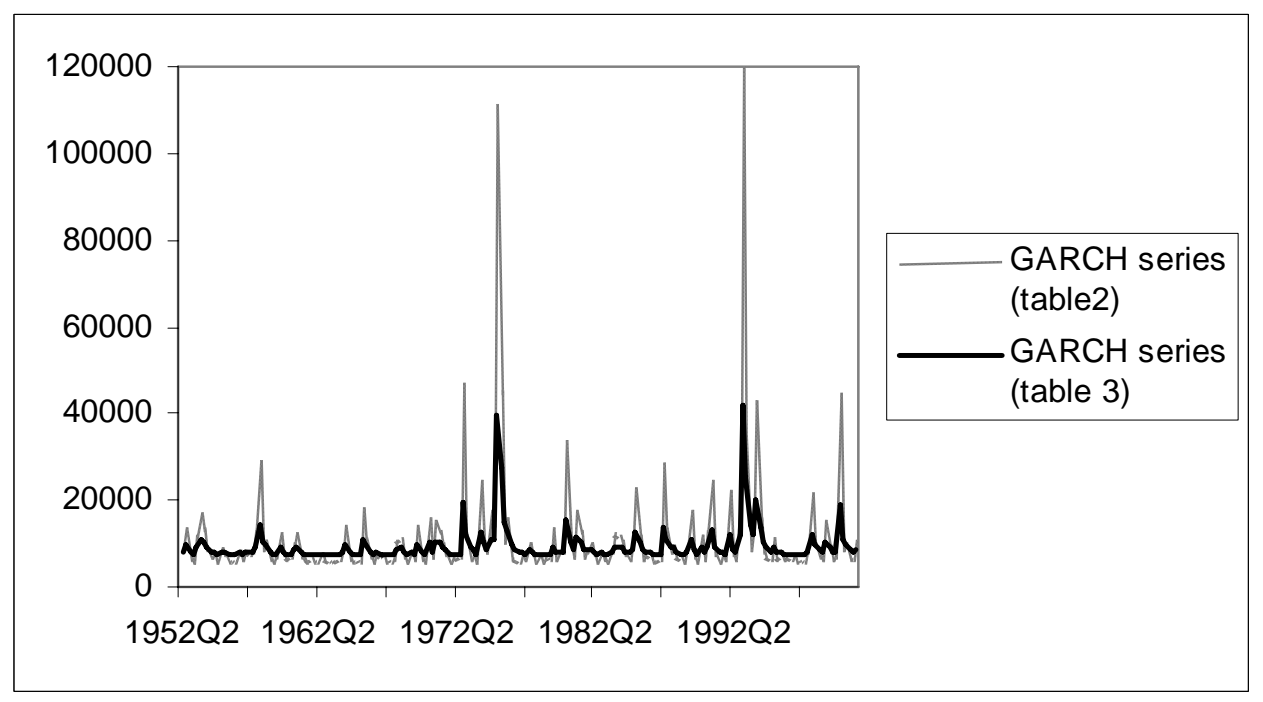


Figure 3: Unobserved state $\psi_{t}$ with $90 \%$ confidence bands (case table 2)

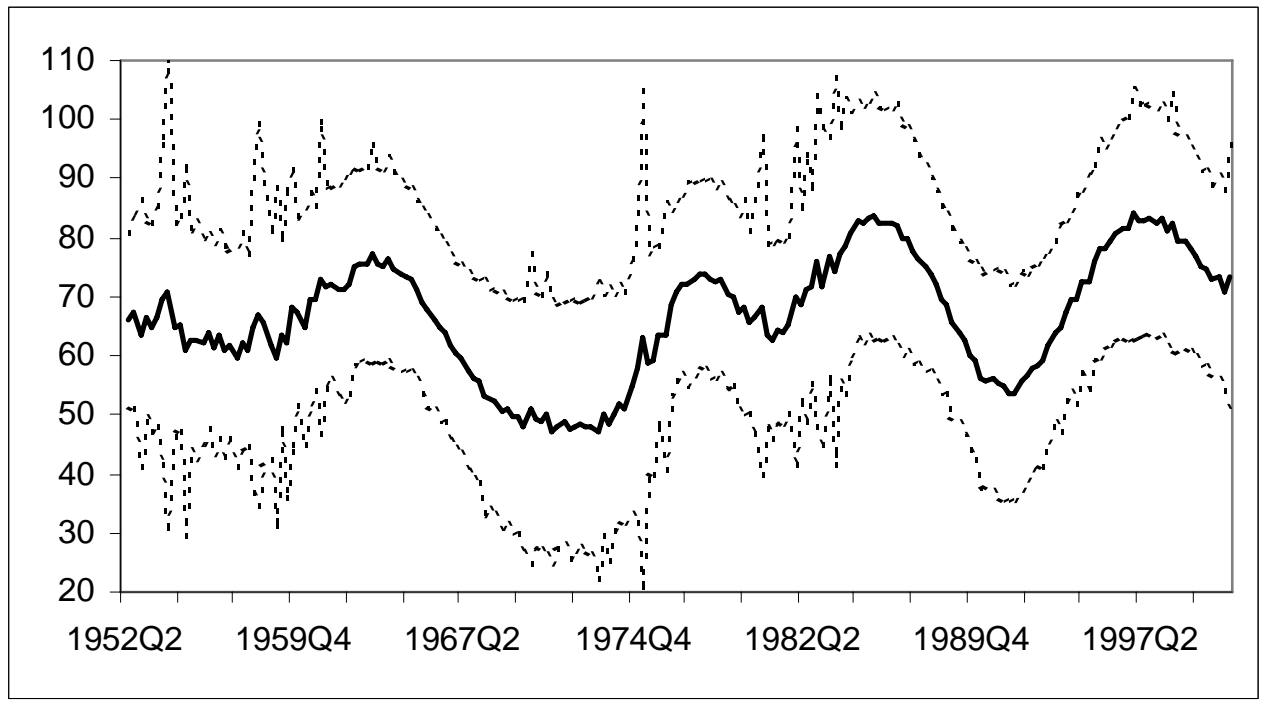

Figure 4: Real per capita consumption changes, trend, and unobserved state $\psi_{t}$

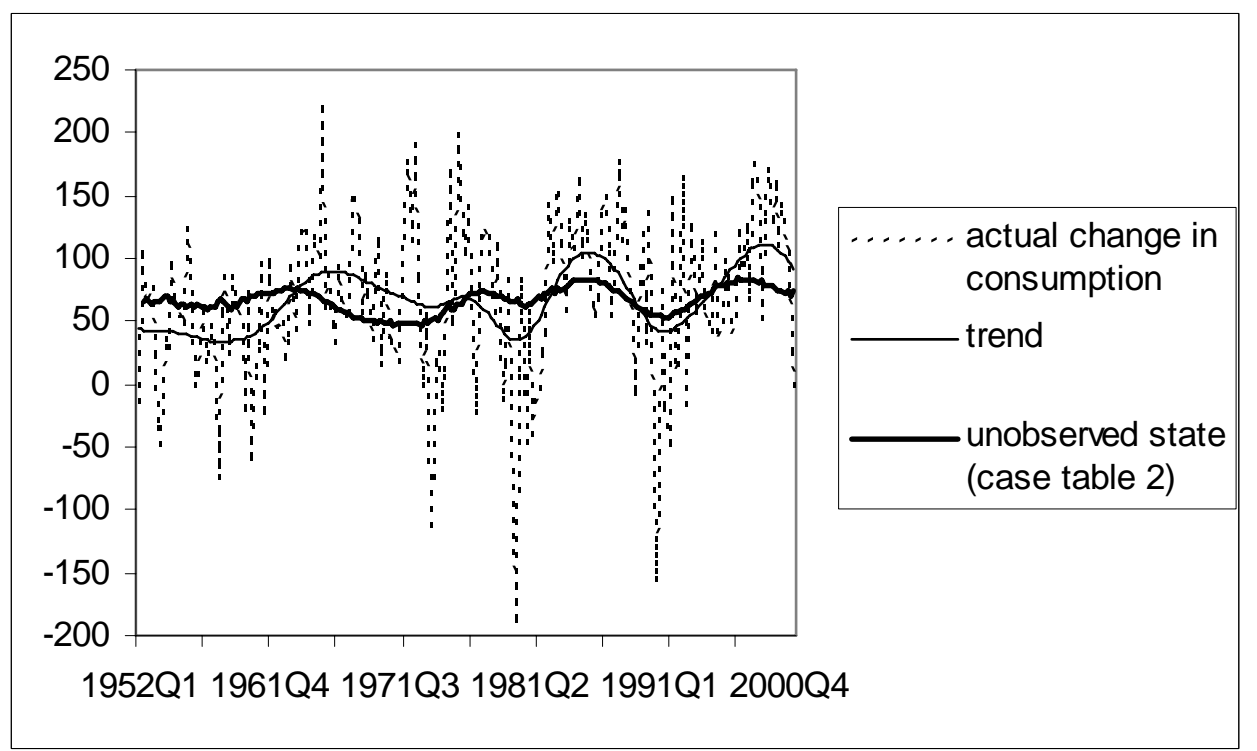


Figure 5: Unobserved state $\psi_{t}$ with $90 \%$ confidence bands (case table 4 )

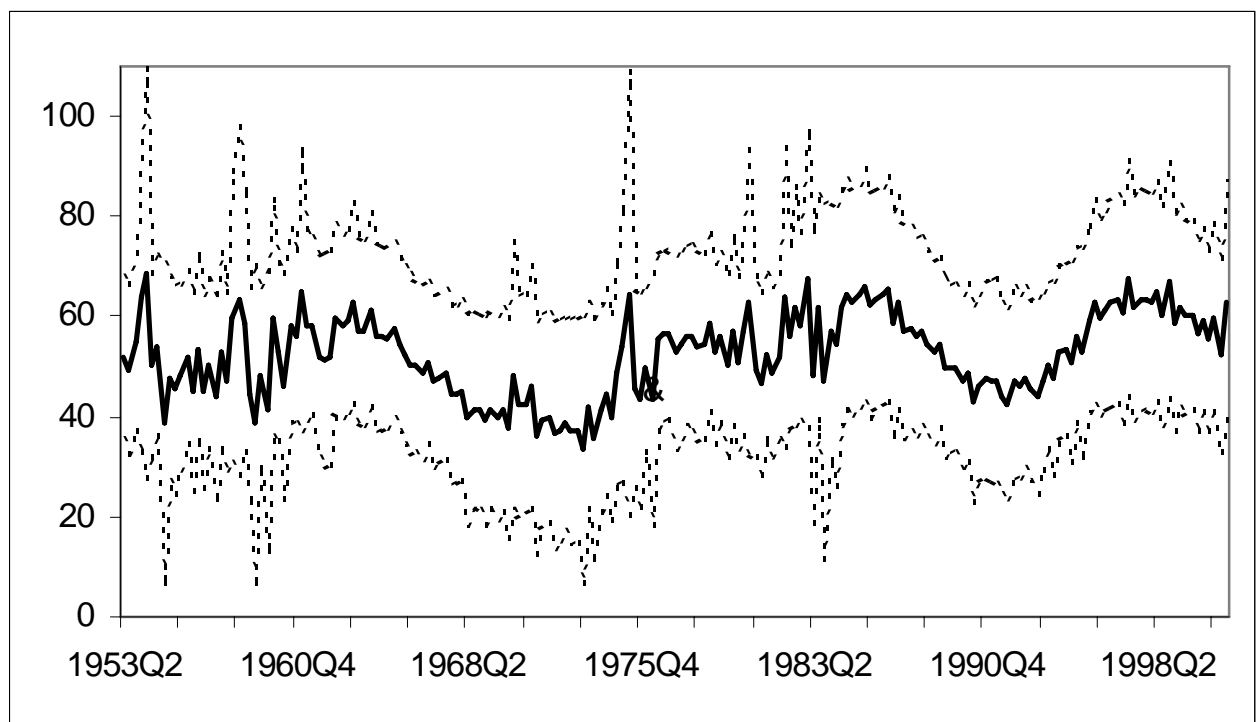

Figure 6: Real per capita consumption changes, trend, and unobserved state $\psi_{t}$

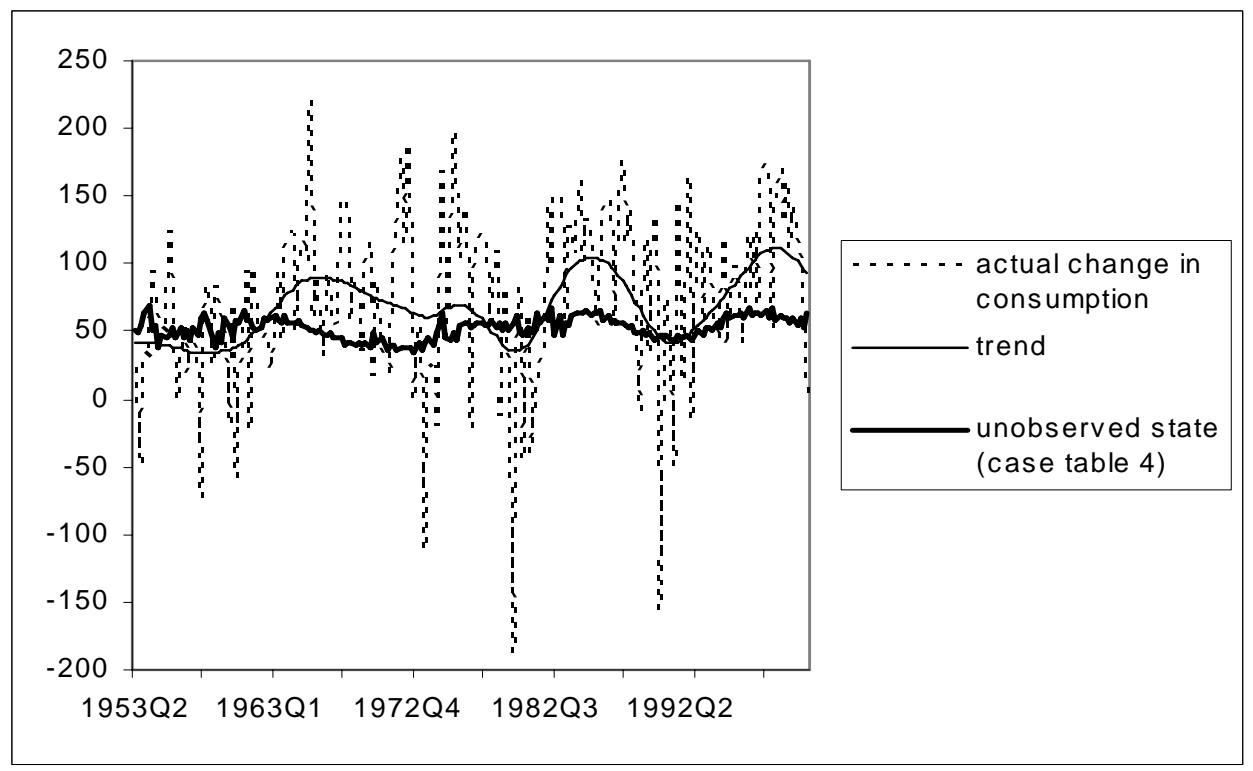

